\title{
Results and techniques for higher order calculations within the gradient-flow formalism
}

\author{
Johannes Artz, ${ }^{a}$ Robert V. Harlander, ${ }^{a}$ Fabian Lange, ${ }^{a}$ Tobias Neumann ${ }^{b, c}$ \\ and Mario Prausa ${ }^{d}$ \\ ${ }^{a}$ Institute for Theoretical Particle Physics and Cosmology, RWTH Aachen University, \\ D-52056 Aachen, Germany \\ ${ }^{b}$ Department of Physics, Illinois Institute of Technology, \\ Chicago, Illinois 60616, U.S.A. \\ ${ }^{c}$ Fermilab, \\ PO Box 500, Batavia, Illinois 60510, U.S.A. \\ ${ }^{d}$ Physikalisches Institut, Albert-Ludwigs-Universität, \\ D-79085 Freiburg, Germany \\ E-mail: johannes.artz@rwth-aachen.de, \\ harlander@physik.rwth-aachen.de, flange@physik.rwth-aachen.de, \\ tneumann@fnal.gov, mario.prausa@physik.uni-freiburg.de
}

ABSTRACT: We describe in detail the implementation of a systematic perturbative approach to observables in the QCD gradient-flow formalism. This includes a collection of all relevant Feynman rules of the five-dimensional field theory and the composite operators considered in this paper. Tools from standard perturbative calculations are used to obtain Green's functions at finite flow time $t$ at higher orders in perturbation theory. The three-loop results for the quark condensate at finite $t$ and the conversion factor for the "ringed" quark fields to the $\overline{\mathrm{MS}}$ scheme are presented as applications. We also re-evaluate an earlier result for the three-loop gluon condensate, improving on its accuracy.

KeYwords: Lattice QCD, Perturbative QCD

ARXIV EPRINT: 1905.00882 


\section{Contents}

1 Introduction 1

2 The QCD gradient flow in perturbation theory 2

2.1 Definition of the gradient flow 3

2.2 Perturbative solution of the flow equations 5

$\begin{array}{lll}2.3 & \text { Feynman rules } & 6\end{array}$

3 Automated implementation $\quad 11$

3.1 Generation of Feynman diagram expressions 11

$\begin{array}{lll}3.2 & \text { IBP reduction of flow-time loop integrals } & 12\end{array}$

$\begin{array}{lll}3.3 & \text { Numerical computation of flow-time loop integrals } & 14\end{array}$

4 Observables $\quad \mathbf{1 5}$

$\begin{array}{lll}4.1 & \text { Results for the gluon condensate at three loops } & 17\end{array}$

$\begin{array}{lll}4.2 & \text { Results for the quark condensate at three loops } & 19\end{array}$

5 Gradient-flow coupling and mass $\quad 21$

5.1 Gradient-flow coupling 21

5.2 Gradient-flow mass 22

6 Conclusions 23

$\begin{array}{ll}\text { A Feynman rules } & 24\end{array}$

B Analytical results $\quad 30$

\section{Introduction}

The gradient flow in field theory has proven to be a very useful concept. Originally introduced as a method to regularize divergences and ultraviolet fluctuations in lattice calculations $[1,2]$, it has mainly gained traction through its use for scale-setting in lattice QCD [3-5] and has meanwhile become an indispensable tool in many practical calculations in this field (see, e.g. refs. [6-9]). While first focused on QCD, generalizations of the gradient-flow formalism (GFF) yield a much wider range of applications, for example the study of dualities in field theory [10-15].

In QCD, the formulation as a five-dimensional field theory has been presented in ref. [16], where the additional dimension is associated with the so-called flow time. The form of the fundamental theory (i.e., actual QCD) serves as the boundary condition at vanishing flow time. It has been proven that, when expressed in terms of renormalized 
parameters and fields, composite operators at positive flow time are finite [16]. This property and the realization that the gradient flow can be used as a matching scheme between lattice and perturbative calculations shed light on a whole new set of applications that are currently being explored.

One of the first possible cross-fertilizations among perturbative and lattice QCD is given by the definition of a new scheme for the strong coupling, defined by the gluon condensate (QCD action density) at finite flow time [3]. Its perturbative relation to the $\overline{\mathrm{MS}}$ coupling is known through three loops [17]. ${ }^{1}$ Another cross-boundary application is the gradient-flow definition of the energy-momentum tensor, which uses the small-flow-time expansion in order to express it in terms of well-defined composite operators at positive flow time and perturbatively accessible coefficient functions [19-22]. Yet another example is the proposal to relate Euclidean quasi $\mathrm{PDFs}^{2}$ on the lattice to perturbative light-front PDFs by using the gradient flow [23, 24].

All these applications rely on input from the lattice as well as from perturbative calculations, both at finite flow time. It was found that higher order corrections in perturbation theory are crucial for reducing the perturbative truncation error as estimated by the variation of the renormalization scale $[17,21]$. Such corrections were also found to significantly stabilize the required extrapolations of the corresponding lattice results [22].

Despite the fact that loop integrals in the GFF involve additional exponential factors as well as integrations over flow-time variables, many important techniques for regular perturbative calculations retain their usefulness, albeit in slightly generalized form. It is the goal of this paper to provide the basis for further perturbative calculations within the GFF, and thus to contribute to the further exploration of the capabilities of this approach. Section 2 reviews the QCD gradient flow in perturbation theory, recapitulating results of refs. $[3,6,16]$. The various stages of automation in a perturbative multi-loop calculation of correlation functions are described in section 3: the generation of Feynman diagrams and insertion and evaluation of Feynman rules, followed by a reduction of the loop integrals to a set of master integrals, and finally the numerical evaluation of the latter. As an application, section 4 presents the three-loop results for the gluon condensate, obtained before in ref. [17], the quark condensate, as well as the "ringed" quark field renormalization constant, which are new results. These quantities allow one to define a precision gradient-flow coupling and gradient-flow mass scheme and their matching to $\overline{\mathrm{MS}}$ as shown in section 5. Our conclusions are presented in section 6 .

\section{The QCD gradient flow in perturbation theory}

For completeness, we collect the main steps of the original derivations of refs. [3, 16] in this section.

\footnotetext{
${ }^{1}$ Unless stated otherwise, we refer to perturbative calculations as performed at infinite volume in this paper. The inclusion of finite-volume effects requires different techniques, such as Numerical Stochastic Perturbation Theory, see ref. [18].

${ }^{2} \mathrm{PDF}=$ parton distribution function.
} 


\subsection{Definition of the gradient flow}

In the following, we work in $D$-dimensional Euclidean space-time with $D=4-2 \epsilon$. The GFF continues the gluon and quark fields $A_{\mu}^{a}(x)$ and $\psi_{\alpha}^{i}(x)$ of $\operatorname{regular}^{3}$ QCD to $(D+1)$ dimensional fields $B_{\mu}^{a}(t, x)$ and $\chi_{\alpha}^{i}(t, x)$ through the boundary conditions

$$
B_{\mu}^{a}(t=0, x)=A_{\mu}^{a}(x), \quad \chi_{\alpha}^{i}(t=0, x)=\psi_{\alpha}^{i}(x)
$$

and the flow equations $[3,6]$

$$
\begin{aligned}
\partial_{t} B_{\mu}^{a} & =\mathcal{D}_{\nu}^{a b} G_{\nu \mu}^{b}+\kappa \mathcal{D}_{\mu}^{a b} \partial_{\nu} B_{\nu}^{b} \\
\partial_{t} \chi & =\Delta \chi-\kappa \partial_{\mu} B_{\mu}^{a} T^{a} \chi \\
\partial_{t} \bar{\chi} & =\bar{\chi} \overleftarrow{\Delta}+\kappa \bar{\chi} \partial_{\mu} B_{\mu}^{a} T^{a}
\end{aligned}
$$

where the "flow time" $t$ is a parameter of mass dimension minus two, and $\kappa$ is a gauge parameter which drops out of physical observables (see below).

The $(D+1)$-dimensional field-strength tensor is defined as

$$
G_{\mu \nu}^{a}=\partial_{\mu} B_{\nu}^{a}-\partial_{\nu} B_{\mu}^{a}+f^{a b c} B_{\mu}^{b} B_{\nu}^{c},
$$

the covariant derivative in the adjoint representation is given by

$$
\mathcal{D}_{\mu}^{a b}=\delta^{a b} \partial_{\mu}-f^{a b c} B_{\mu}^{c},
$$

and

$$
\Delta=\mathcal{D}_{\mu}^{\mathrm{F}} \mathcal{D}_{\mu}^{\mathrm{F}}, \quad \overleftarrow{\Delta}=\overleftarrow{\mathcal{D}}_{\mu}^{\mathrm{F}} \overleftarrow{\mathcal{D}}_{\mu}^{\mathrm{F}}
$$

with the covariant derivative in the fundamental representation,

$$
\mathcal{D}_{\mu}^{\mathrm{F}}=\partial_{\mu}+B_{\mu}^{a} T^{a}, \quad \overleftarrow{\mathcal{D}}_{\mu}^{\mathrm{F}}=\overleftarrow{\partial}_{\mu}-B_{\mu}^{a} T^{a}
$$

As usual, the color indices of the adjoint representation are denoted by $a, b, c, \ldots$, while $\mu, \nu, \rho, \ldots$ are $D$-dimensional Lorentz indices. Color indices of the fundamental representation are denoted by $i, j, k, \ldots$, but they are suppressed throughout this paper, unless required by clarity; similarly for spinor indices $\alpha, \beta, \gamma, \ldots$. The symmetry generators $T^{a}$ are understood in the fundamental representation. The obey the commutation relation

$$
\left[T^{a}, T^{b}\right]=f^{a b c} T^{c}
$$

with the structure constants $f^{a b c}$ and the trace is normalized to

$$
\operatorname{Tr}\left(T^{a} T^{b}\right)=-T_{\mathrm{R}} \delta^{a b}
$$

with $T_{\mathrm{R}}>0$.

\footnotetext{
${ }^{3}$ We use the terms "flowed" and "regular" QCD to distinguish quantities defined at $t>0$ from those defined at $t=0$.
} 
Different choices of $\kappa$ in eq. (2.2) correspond to gauge transformations of the form

$$
\chi \rightarrow \chi^{\prime}=\Lambda \chi \quad \text { and } \quad B_{\mu}^{a} T^{a} \rightarrow B_{\mu}^{a \prime} T^{a}=\Lambda B_{\mu}^{a} T^{a} \Lambda^{-1}+\Lambda \partial_{\mu} \Lambda^{-1},
$$

where

$$
\Lambda(t, x)=e^{-\int_{0}^{t} \mathrm{~d} s \kappa \partial_{\mu} B_{\mu}^{a}(s, x) T^{a}} .
$$

Of course, all observables are independent of the gauge parameter $\kappa$ [3]. In perturbative calculations, it is usually most convenient to set $\kappa=1$.

The flow equations (2.2) can be incorporated in a Lagrangian formalism by defining

$$
\mathcal{L}=\mathcal{L}_{\mathrm{QCD}}+\mathcal{L}_{\text {gauge-fixing }}+\mathcal{L}_{\text {ghost }}+\mathcal{L}_{B}+\mathcal{L}_{\chi}
$$

The first three terms constitute the regular Yang-Mills Lagrangian, with fermions added in the fundamental representation (quarks). Introducing an index $f$ in order to distinguish different quark flavors of mass $m_{f}$, the classical, gauge-fixing, and Faddeev-Popov ghost part are given by

$$
\begin{aligned}
\mathcal{L}_{\mathrm{QCD}} & =\frac{1}{4 g^{2}} F_{\mu \nu}^{a} F_{\mu \nu}^{a}+\sum_{f=1}^{n_{\mathrm{F}}} \bar{\psi}_{f}\left(\not D^{\mathrm{F}}+m_{f}\right) \psi_{f}, \\
\mathcal{L}_{\text {gauge-fixing }} & =\frac{1}{2 g^{2} \xi}\left(\partial_{\mu} A_{\mu}^{a}\right)^{2}, \\
\mathcal{L}_{\text {ghost }} & =\frac{1}{g^{2}} \partial_{\mu} \bar{c}^{a} D_{\mu}^{a b} c^{b},
\end{aligned}
$$

respectively, where

$$
F_{\mu \nu}^{a}=\partial_{\mu} A_{\nu}^{a}-\partial_{\nu} A_{\mu}^{a}+f^{a b c} A_{\mu}^{b} A_{\nu}^{c}
$$

is the regular field strength tensor and

$$
D_{\mu}^{\mathrm{F}}=\partial_{\mu}+A_{\mu}^{a} T^{a}, \quad D_{\mu}^{a b}=\delta^{a b} \partial_{\mu}-f^{a b c} A_{\mu}^{c}
$$

are the regular covariant derivatives in the fundamental and adjoint representation, respectively, $g$ is the gauge coupling, $\xi$ the QCD gauge parameter, and $n_{\mathrm{F}}$ the number of different quark flavors. The flow equations are incorporated by introducing Lagrange multiplier fields

$$
L_{\mu}^{a}(t, x) \quad \text { and } \quad \lambda_{f}(t, x), \bar{\lambda}_{f}(t, x),
$$

of mass dimensions 3 and $5 / 2$ that otherwise carry the same quantum numbers as the flowed gluon and quark/antiquark fields $B_{\mu}^{a}$ and $\chi, \bar{\chi}$, respectively. Their Euler-Lagrange equations derived from

$$
\begin{aligned}
& \mathcal{L}_{B}=-2 \int_{0}^{\infty} \mathrm{d} t \operatorname{Tr}\left[L_{\mu}^{a} T^{a}\left(\partial_{t} B_{\mu}^{b} T^{b}-\mathcal{D}_{\nu}^{b c} G_{\nu \mu}^{c} T^{b}-\kappa \mathcal{D}_{\mu}^{b c} \partial_{\nu} B_{\nu}^{c} T^{b}\right)\right] \\
& \mathcal{L}_{\chi}=\sum_{f=1}^{n_{\mathrm{F}}} \int_{0}^{\infty} \mathrm{d} t\left(\bar{\lambda}_{f}\left(\partial_{t}-\Delta+\kappa\left(\partial_{\mu} B_{\mu}^{a}\right) T^{a}\right) \chi_{f}+\bar{\chi}_{f}\left(\overleftarrow{\partial_{t}}-\overleftarrow{\Delta}-\kappa\left(\partial_{\mu} B_{\mu}^{a}\right) T^{a}\right) \lambda_{f}\right)
\end{aligned}
$$

indeed lead to eq. (2.2) [16]. 
Note that the Lagrangian (2.11) does not include flowed ghost fields $d^{a}(t, x)$ and $\bar{d}^{a}(t, x)$. They arise in the same way as the usual Faddeev-Popov ghosts $c^{a}(x)$ and $\bar{c}^{a}(x)$ due to gauge-fixing, and obey the initial condition

$$
\left.d^{a}(t, x)\right|_{t=0}=c^{a}(x) .
$$

Similar to the ghosts of the regular gauge fields, they always form closed loops as long as there are no external $d^{a}$ or $\bar{d}^{a}$ fields (which can be avoided by considering only physical degrees of freedom in amplitudes with external gluons). As becomes clear later, closed loops of only flowed fields vanish, so that one can omit $d^{a}$ and $\bar{d}^{a}$ already at the level of the Lagrangian.

\subsection{Perturbative solution of the flow equations}

Let us introduce the short-hand notation

$$
\int_{p} \equiv \int \frac{\mathrm{d}^{D} p}{(2 \pi)^{D}}, \quad \int_{x} \equiv \int \mathrm{d}^{D} x
$$

where it should be clear from the context whether an integration variable is in position $(x, y, z, \ldots)$ or momentum space $(p, k, q, \ldots)$.

It is helpful to separate the flow equation (2.2) of the flowed gauge field $B_{\mu}^{a}(t, x)$ into a linear and a non-linear part:

$$
\begin{aligned}
\partial_{t} B_{\mu}^{a} & =\partial_{\nu} \partial_{\nu} B_{\mu}^{a}+(\kappa-1) \partial_{\mu} \partial_{\nu} B_{\nu}^{a}+R_{\mu}^{a}, \\
R_{\mu}^{a} & =2 f^{a b c} B_{\nu}^{b} \partial_{\nu} B_{\mu}^{c}-f^{a b c} B_{\nu}^{b} \partial_{\mu} B_{\nu}^{c}+(\kappa-1) f^{a b c} B_{\mu}^{b} \partial_{\nu} B_{\nu}^{c}+f^{a b e} f^{c d e} B_{\nu}^{b} B_{\nu}^{c} B_{\mu}^{d} .
\end{aligned}
$$

The linear equation can be solved by introducing the integration kernel

$$
K_{\mu \nu}(t, x)=\int_{p} \frac{e^{\mathrm{i} p x}}{p^{2}}\left(\left(\delta_{\mu \nu} p^{2}-p_{\mu} p_{\nu}\right) e^{-t p^{2}}+p_{\mu} p_{\nu} e^{-\kappa t p^{2}}\right) \equiv \int_{p} e^{\mathrm{i} p x} \widetilde{K}_{\mu \nu}(t, p),
$$

which fulfills

$$
\lim _{t \rightarrow 0} K_{\mu \nu}(t, x)=\delta_{\mu \nu} \delta^{(D)}(x) .
$$

Taking into account the initial condition (2.1), the full solution of the flow equation is then given by

$$
B_{\mu}^{a}(t, x)=\int_{y} K_{\mu \nu}(t, x-y) A_{\nu}^{a}(y)+\int_{y} \int_{0}^{t} \mathrm{~d} s K_{\mu \nu}(t-s, x-y) R_{\nu}^{a}(s, y),
$$

or, in momentum space,

$$
\widetilde{B}_{\mu}^{a}(t, p)=\int_{x} e^{-\mathrm{i} p x} B_{\mu}^{a}(t, x)=\widetilde{K}_{\mu \nu}(t, p) \widetilde{A}_{\nu}^{a}(p)+\int_{0}^{t} \mathrm{~d} s \widetilde{K}_{\mu \nu}(t-s, p) \widetilde{R}_{\nu}^{a}(s, p) .
$$

By inserting the solution iteratively into itself, one can express the Fourier transform of the non-linear part of eq. (2.19) as

$$
\begin{array}{r}
\widetilde{R}_{\mu}^{a}(t, p)=\int_{q, l, k}(2 \pi)^{D} \delta^{(D)}(p-q-l-k)\left[\delta^{(D)}(k) \cdot X_{2, \mu \nu \rho}^{a b c}(q, l) \widetilde{B}_{\nu}^{b}(t, q) \widetilde{B}_{\rho}^{c}(t, l)\right. \\
\left.+X_{3, \mu \nu \rho \sigma}^{a b c d} \widetilde{B}_{\nu}^{b}(t, q) \widetilde{B}_{\rho}^{c}(t, l) \widetilde{B}_{\sigma}^{d}(t, k)\right]
\end{array}
$$


where

$$
\begin{aligned}
X_{2, \mu \nu \rho}^{a b c}(q, l)= & -\mathrm{i} f^{a b c}\left[(l-q)_{\mu} \delta_{\nu \rho}+2 q_{\rho} \delta_{\mu \nu}-2 l_{\nu} \delta_{\mu \rho}+(\kappa-1)\left(q_{\nu} \delta_{\mu \rho}-l_{\rho} \delta_{\mu \nu}\right)\right], \\
X_{3, \mu \nu \rho \sigma}^{a b c d}= & f^{a b e} f^{c d e}\left(\delta_{\mu \sigma} \delta_{\nu \rho}-\delta_{\mu \rho} \delta_{\nu \sigma}\right)+f^{a d e} f^{b c e}\left(\delta_{\mu \rho} \delta_{\nu \sigma}-\delta_{\mu \nu} \delta_{\rho \sigma}\right) \\
& +f^{a c e} f^{d b e}\left(\delta_{\mu \nu} \delta_{\rho \sigma}-\delta_{\mu \sigma} \delta_{\nu \rho}\right) .
\end{aligned}
$$

The structure of $X_{3}$ is identical to the four-gluon vertex of regular QCD. When formulating the Feynman rules later, $X_{2}$ and $X_{3}$ describe the three- and four-point vertices of the flowed gluon fields.

The flow equation (2.2) for the flowed quark fields can be solved by again splitting it into a linear and a non-linear part,

$$
\partial_{t} \chi=\partial_{\mu} \partial_{\mu} \chi+\Delta^{\prime} \chi \quad \text { with } \quad \Delta^{\prime}=(1-\kappa) \partial_{\mu} B_{\mu}^{a} T^{a}+2 B_{\mu}^{a} T^{a} \partial_{\mu}+B_{\mu}^{a} B_{\mu}^{b} T^{a} T^{b} .
$$

The linear equation is solved by the integration kernel

$$
K(t, x)=\int_{p} e^{\mathrm{i} p x} e^{-t p^{2}} \equiv \int_{p} e^{\mathrm{i} p x} \widetilde{K}(t, p),
$$

with the help of which we can write the full solution as

$$
\chi(t, x)=\int_{y} K(t, x-y) \psi(y)+\int_{y} \int_{0}^{t} \mathrm{~d} s K(t-s, x-y) \Delta^{\prime} \chi(s, y) .
$$

Here and in what follows, we suppress the flavor index $f$ unless required for clarity. The non-linear part of the Fourier-transformed field

$$
\widetilde{\chi}(t, p)=\widetilde{K}(t, p) \widetilde{\psi}(p)+\int_{0}^{t} \mathrm{~d} s \widetilde{K}(t-s, p) \widetilde{\Delta^{\prime} \chi}(s, p)
$$

can be expressed as

$$
\begin{aligned}
\widetilde{\Delta^{\prime} \chi}(t, p)=\int_{q, r}(2 \pi)^{D} \delta^{(D)}(p-q-l-r) & {\left[\delta^{(D)}(l) \cdot Y_{1, \nu}^{b}(p, q, r) \widetilde{B}_{\nu}^{b}(t, q)\right.} \\
& \left.+\frac{1}{2} Y_{2, \nu \rho}^{b c}(p, q, l, r) \widetilde{B}_{\nu}^{b}(t, q) \widetilde{B}_{\rho}^{c}(t, l)\right] \widetilde{\chi}(t, r),
\end{aligned}
$$

where

$$
Y_{1, \nu}^{b}(q, r)=\mathrm{i}\left(2 r_{\nu}+(1-\kappa) q_{\nu}\right) T^{b}, \quad Y_{2, \nu \rho}^{b c}=\delta_{\nu \rho}\left\{T^{b}, T^{c}\right\} .
$$

These expressions lead to the three- and four-point vertices of the flowed quark fields. For $\bar{\chi}$ one proceeds analogously.

\subsection{Feynman rules}

(Flowed) propagators. Plugging in the solution of the flowed gluon field in momentum space eq. (2.23) into the two-point function, one finds [16]

$$
\begin{aligned}
\left.\left\langle\widetilde{B}_{\mu}^{a}(t, p) \widetilde{B}_{\nu}^{b}(s, q)\right\rangle\right|_{\mathrm{LO}} & =\widetilde{K}_{\mu \rho}(t, p) \widetilde{K}_{\nu \sigma}(s, q)\left\langle\widetilde{A}_{\rho}^{a}(p) \widetilde{A}_{\sigma}^{b}(q)\right\rangle \\
& =(2 \pi)^{D} \delta^{(D)}(p+q) g^{2} D_{\mu \nu}^{a b}(p, t+s, \xi, \kappa),
\end{aligned}
$$


where

$$
D_{\mu \nu}^{a b}(p, t, \xi, \kappa)=\delta^{a b} \frac{1}{p^{2}}\left(\left(\delta_{\mu \nu}-\frac{p_{\mu} p_{\nu}}{p^{2}}\right) e^{-t p^{2}}+\xi \frac{p_{\mu} p_{\nu}}{p^{2}} e^{-\kappa t p^{2}}\right)
$$

and we have used the result for the fundamental gluon propagator,

$$
\left.\left\langle\widetilde{A}_{\mu}^{a}(p) \widetilde{A}_{\nu}^{b}(q)\right\rangle\right|_{\mathrm{LO}}=(2 \pi)^{D} \delta^{(D)}(p+q) g^{2} D_{\mu \nu}^{a b}(p, 0, \xi, 0) .
$$

The factor $g^{2}$ is taken into account in the corresponding vertices further below. Since the flowed gluon propagator coincides with the fundamental gluon propagator at $t+s=0$, we can express both of them by the same Feynman rule:

$$
\begin{gathered}
p \\
s, \nu, b \text { unuen- } t, \mu, a=D_{\mu \nu}^{a b}(p, t+s, \xi, \kappa) .
\end{gathered}
$$

We refer to this as the (flowed) gluon propagator. Eq. (2.35) also applies to the mixed propagator $\langle\widetilde{A} \widetilde{B}\rangle$, which is obtained by setting one of the two flow-time variables to zero.

The same can be done for flowed quark fields. Inserting their momentum space solution in eq. (2.29) into the two-point function results in

$$
\begin{aligned}
\left.\left\langle\widetilde{\chi}_{\alpha}^{i}(t, p) \widetilde{\bar{\chi}}_{\beta}^{j}(s, q)\right\rangle\right|_{\mathrm{LO}} & =\widetilde{K}(t, p) \widetilde{K}(s, q)\left\langle\widetilde{\psi}_{\alpha}^{i}(p) \widetilde{\bar{\psi}}_{\beta}^{j}(q)\right\rangle \\
& =(2 \pi)^{D} \delta^{(D)}(p+q) S_{\mathrm{F}, \alpha \beta}^{i j}(p, m, t+s),
\end{aligned}
$$

where

$$
S_{\mathrm{F}}^{i j}(p, m, t)=\delta^{i j} \frac{-i \not p+m}{p^{2}+m^{2}} e^{-t p^{2}},
$$

and we have used the result for the fundamental quark propagator

$$
\left\langle\widetilde{\psi}_{\alpha}^{i}(p) \widetilde{\bar{\psi}}_{\beta}^{j}(q)\right\rangle=(2 \pi)^{D} \delta^{(D)}(p+q) S_{\mathrm{F}, \alpha \beta}^{i j}(p, m, 0) .
$$

Since one can express the fundamental propagator through the flowed propagator at vanishing flow times, both can be represented by the same Feynman rule:

$$
s, \beta, j \longrightarrow \stackrel{p}{\longrightarrow} t, \alpha, i=S_{\mathrm{F}, \alpha \beta}^{i j}(p, m, t+s) .
$$

We refer to this as the (flowed) quark propagator, which again includes the mixed propagators $\langle\psi \bar{\chi}\rangle$ and $\langle\chi \bar{\psi}\rangle$.

Flow lines. Since there are no quadratic terms of the Lagrange multiplier fields $L_{\mu}^{a}, \lambda$, and $\bar{\lambda}$ in eq. (2.16), there are no propagators for these fields. However, the Lagrangian contains bilinear terms of a flowed field and a Lagrange multiplier field. Using standard methods, one derives the two-point function at leading order as

$$
\left\langle B_{\mu}^{a}(t, x) L_{\nu}^{b}(s, y)\right\rangle=\delta^{a b} H_{\mu \nu}(t-s, x-y),
$$


where $H_{\mu \nu}(t, x)$ obeys the equation

$$
\left[\left(\partial_{t}-\partial_{\sigma} \partial_{\sigma}\right) \delta_{\mu \nu}+(1-\kappa) \partial_{\mu} \partial_{\nu}\right] H_{\nu \rho}(t, x)=\frac{1}{2 T_{\mathrm{R}}} \delta_{\mu \rho} \delta(t) \delta^{(D)}(x),
$$

and $T_{\mathrm{R}}$ is the usual color trace normalization. Since the fundamental gluon field $A_{\mu}^{a}(x)=$ $B_{\mu}^{a}(0, x)$ does not couple to the Lagrange multiplier field $L_{\mu}^{a}$, and all flow-time variables are positive, one can impose the initial condition

$$
\left.\left\langle B_{\mu}^{a}(t, x) L_{\nu}^{b}(s, y)\right\rangle\right|_{t=0}=0 \quad \Rightarrow \quad H_{\mu \nu}(-s, x)=0,
$$

so that the unique solution becomes

$$
H_{\mu \nu}(t, x)=\frac{1}{2 T_{\mathrm{R}}} \theta(t) K_{\mu \nu}(t, x) .
$$

We refer to the $\langle B L\rangle$ bilinear as "gluon flow line". The inverse of the factor $1 /\left(2 T_{\mathrm{R}}\right)$ appears in the corresponding "flow vertices" further below. We can therefore discard it altogether and write

$$
\stackrel{p}{\stackrel{p}{\longrightarrow}} \underset{s, \nu}{\longrightarrow}, \mu, a=\delta^{a b} \theta(t-s) \widetilde{K}_{\mu \nu}(t-s, p),
$$

where $\widetilde{K}_{\mu \nu}(t, p)$ has been defined in eq. (2.20), and the adjacent arrow indicates the direction towards increasing flow time as implied by the $\theta$-distribution. As opposed to an actual propagator, the gluon flow line is a regular function for all $p$.

Similarly, one determines the mixed fermionic two-point function at leading order as

$$
\left\langle\chi_{\alpha}^{i}(t, x) \bar{\lambda}_{\beta}^{j}(s, y)\right\rangle=\delta_{\alpha \beta} \delta^{i j} G(t-s, x-y),
$$

where $G(t, x)$ obeys

$$
\left(\partial_{t}-\partial_{\mu} \partial_{\mu}\right) G(t, x)=\delta(t) \delta^{(D)}(x),
$$

with the condition

$$
\left.\left\langle\chi_{\alpha}^{i}(t, x) \bar{\lambda}_{\beta}^{j}(s, y)\right\rangle\right|_{t=0}=0 \Rightarrow G(-s, x)=0 .
$$

The unique solution reads

$$
G(t, x)=\theta(t) K(t, x) .
$$

The Fourier transformed expression defines the "fermion flow line" Feynman rule, where the $\theta$-distribution again imposes a direction as indicated by the adjacent arrow pointing towards increasing flow time:

$$
s, \beta, j \longrightarrow
$$

where $\widetilde{K}(t, p)$ has been defined in eq. (2.27). As usual, the arrow on the fermion line denotes the "charge flow" of the fermion. In this way, we have a unified Feynman rule for 
both the $\langle\chi \bar{\lambda}\rangle$ and the $\langle\lambda \bar{\chi}\rangle$ bilinear, where the latter can be obtained analogously as above and simply corresponds to reversing the direction of the charge flow:

$$
s, \beta, j \stackrel{p}{\longrightarrow} t, \alpha, i=\delta_{\alpha \beta} \delta_{i j} \theta(t-s) \widetilde{K}(t-s, p) .
$$

Since $\widetilde{K}(t, p)$ only depends on $p$ only quadratically, the momentum direction for the fermion flow lines is irrelevant.

Flow vertices. Since $\mathcal{L}_{B}$ and $\mathcal{L}_{\chi}$ of eq. (2.16) are proportional to a Lagrange multiplier field, the resulting vertices always involve at least one flow line. Such vertices are always associated with a flow-time parameter which is integrated over. We denote them by "flow vertices" and represent them by empty circles in Feynman diagrams. The corresponding Feynman rules can be derived straightforward. In this paper, we define Feynman rules by assuming all momenta to be outgoing.

The three-point gluon flow vertex is governed by $X_{2}$ :

$$
\begin{aligned}
& \nu, b
\end{aligned}
$$

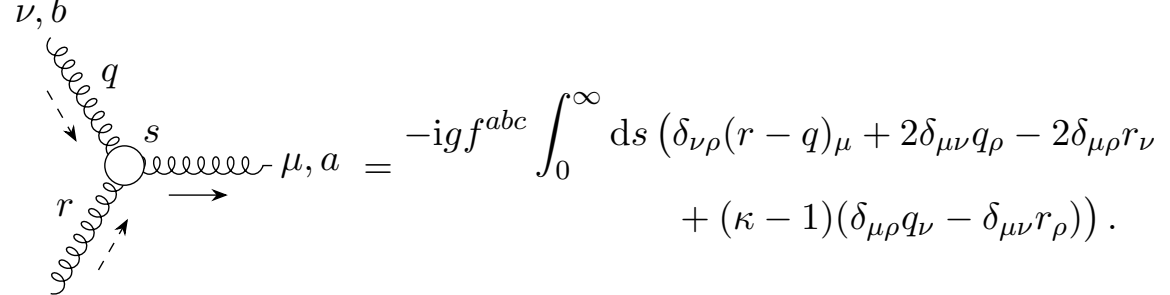

The interaction terms of $\mathcal{L}_{B}$ involve exactly one Lagrange multiplier field $L(s)$, cf. eq. (2.16). According to eqs. (2.42) and (2.44), it must be contracted with a flowed gluon field $B(t)$ at larger flow time $t>s$. Thus, the vertex in eq. (2.51) contains exactly one outgoing flow line. On the other hand, each of the two flowed gluon fields $B(s)$ in the interaction terms of $\mathcal{L}_{B}$ can be contracted either with a Lagrange multiplier field $L\left(t^{\prime}\right)$ at smaller flow time $t^{\prime}<s$, resulting in an ingoing flow line, or with another flowed gluon field $B\left(s^{\prime}\right)$. In this sense, the Feynman rule (2.51) actually represents three vertices, displayed in figure 1: they all contain one outgoing flow line, while each of the other two lines can either be an ingoing flow line or a flowed gluon propagator. ${ }^{4}$ The dashed arrows in eq. (2.51) indicate lines which can be both a flow line or a propagator, while the solid arrows always denote flow lines.

Similarly, the four-point gluon flow vertex is governed by $X_{3}$ :

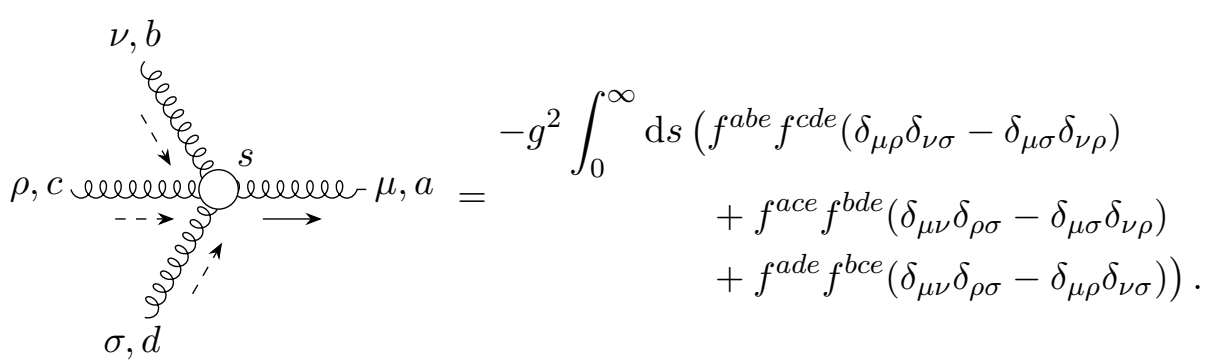

\footnotetext{
${ }^{4}$ We focus on the calculation of Green's functions in this paper; if one calculates amplitudes, the lines could also represent external "particles", of course.
} 

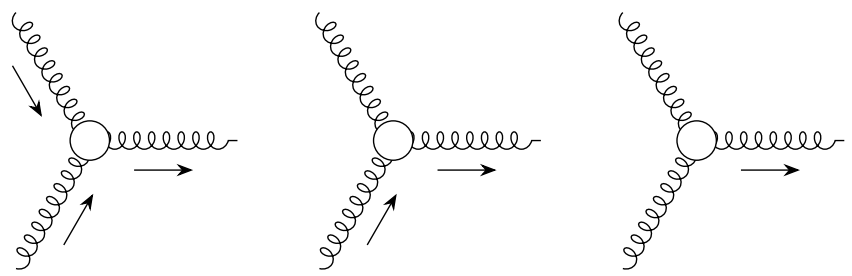

Figure 1. The three versions of the vertex $X_{2}$. Lines with an adjacent arrow denote flow lines, the others are flowed (or regular) gluons.

Again, there is exactly one outgoing flow line, while the other three lines are either flowed gluons or incoming flow lines. This means that the Feynman rule (2.52) actually represents four different vertices.

Note that the factor $2 T_{\mathrm{R}}$, arising from the trace in eq. (2.16), has been discarded in both vertices in accordance with the normalization of eq. (2.44).

Similar considerations applied to $\mathcal{L}_{\chi}$ lead to vertices involving flowed quark fields. For example, the quark flow vertex with one gluon is described by the following Feynman rule:

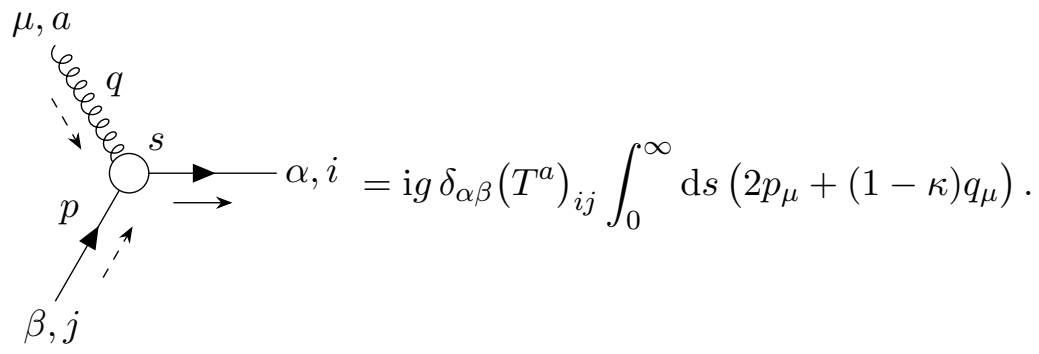

In this case, the fermion line with outgoing charge flow is also outgoing in the gradient flow, while the other two lines can be either flowed propagators, or ingoing flow lines. Thus, this Feynman rule actually represents four different vertices.

A complete list of the Feynman rules can be found in appendix A.

Let us summarize the Feynman rules for the GFF and point out a few more features:

1. Propagators and flow lines always carry an exponential factor $\mathrm{e}^{ \pm t p^{2}}$ for each vertex, where $t$ is the flow time of the vertex.

2. Flow lines always start at a flow vertex. They end either at another flow vertex or an external operator. The $\theta$-distribution $\theta(t-s)$ implies a flow-time direction, which we denote by an adjacent arrow in Feynman diagrams.

3. A flow vertex always has an outgoing flow line connected to it. The other lines are either incoming flow lines, or flowed propagators. Each flow vertex is defined at a flow time $s$ and implies an integration $\int_{0}^{\infty} \mathrm{d} s$. The $\theta$-distribution of the outgoing flow line restricts the integration to a finite upper limit $t$, i.e. $\int_{0}^{t} \mathrm{~d} s$.

4. Diagrams with closed flow-line loops vanish, because the integration interval shrinks to a point. Hence, only diagrams whose flow lines form trees contribute to any observable. 


\section{Automated implementation}

\subsection{Generation of Feynman diagram expressions}

We generate the Feynman diagrams including symmetry factors and signs (from closed fermion loops) with the help of the program qgraf $[25,26]$. In the notation of this program, the propagators for the gluon, the ghost, and a single quark flavor can be defined as

$[g, g,+],[c, C,-],[f q, f Q,-]$.

The sign in the third entry of each square bracket denotes whether the particle is a boson or a fermion.

The flow lines for the gluon and the quark are implemented by introducing separate fields $\mathrm{b}, \mathrm{fr}$, and $\mathrm{fs}$ for $L, \lambda$, and $\bar{\lambda}$, respectively. They are implemented as

$[b, B,+],[f r, f R,-],[f s, f S,-]$,

where the latter two represent the $\langle\chi \bar{\lambda}\rangle$ and $\langle\lambda \bar{\chi}\rangle$ bilinears (see eqs. (2.49) and (2.50)). With these fields, we can then define the regular as well as the flow vertices. For example, for the trilinear flow vertex of the pure gauge theory defined in eq. (2.51), we define

$[B, b, b],[B, b, g],[B, g, g]$,

which corresponds to the three combinations of figure 1 .

Already in regular QCD it is convenient to separate the color structure from the rest of the calculation. This becomes non-trivial in cases which involve the four-gluon vertex. It is thus convenient to introduce an auxiliary "particle" $\Sigma_{\mu \nu}^{a}$ whose "propagator" is given by $[27]$

$$
\rho, \sigma, b========\mu, \nu, a=\delta^{a b} \delta_{\mu \rho} \delta_{\nu \sigma} .
$$

The four-gluon vertex can then be replaced by a trilinear $g g \Sigma$ vertex whose Feynman rule reads

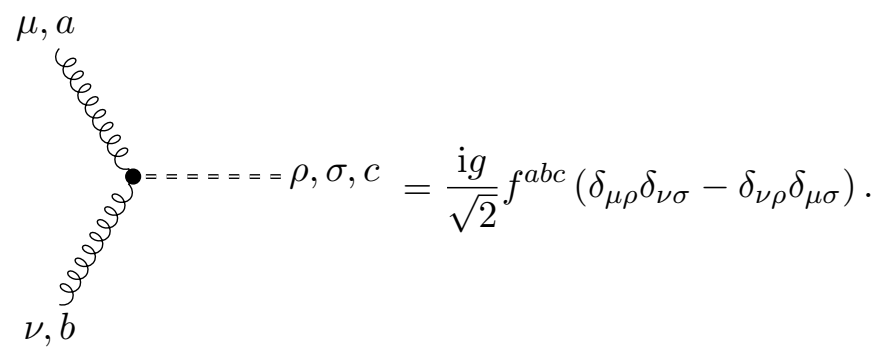

This allows to factorize the color factor off all Feynman diagrams, albeit at the cost of increasing their number. We proceed correspondingly for the quartic gluon flow vertices by introducing an additional $\Sigma$ particle which is directed in flow time. It forms trilinear vertices with gluons and gluon flow lines, as shown explicitly in appendix A. Again, they contain exactly one outgoing ( $\Sigma$ or gluon) flow line. 
By default, qgraf also generates diagrams with closed flow-line loops. We use a Perl script to parse the qgraf output in order to eliminate such diagrams before the actual calculation. They vanish trivially algebraically though, as discussed earlier. In the same way we multiply diagrams by a factor $n_{\mathrm{F}}$ for each closed fermion loop. We then process the diagrams with the help of q2e/exp [28, 29] in order to insert the Feynman rules and convert the diagrams to FORM code.

Within FORM [30, 31], we contract the Lorentz indices, take the fermion traces, and simplify the resulting expressions to a standard form of scalar integrals, as defined below. The color factor is evaluated separately with the help of the color package [32].

For example at the three-loop level (as needed for the results in this paper) the flowtime integrals can be described in the following form

$$
I(t, \mathbf{n}, \mathbf{a}, \mathbf{c}, D)=\left(\prod_{r=1}^{N} \int_{0}^{t_{r}^{\mathrm{up}}} \mathrm{d} t_{r} t_{r}^{c_{r}}\right) \int_{p_{1}, p_{2}, p_{3}} \frac{\exp \left[\sum_{k, i, j} a_{k i j} t_{k} p_{i} \cdot p_{j}\right]}{p_{1}^{2 n_{1}} p_{2}^{2 n_{2}} p_{3}^{2 n_{3}} p_{4}^{2 n_{4}} p_{5}^{2 n_{5}} p_{6}^{2 n_{6}}},
$$

where

$$
\begin{aligned}
& \mathbf{n}=\left\{n_{1}, \ldots, n_{6}\right\}, \quad \mathbf{c}=\left\{c_{1}, \ldots, c_{N}\right\}, \\
& \mathbf{a}=\left\{a_{k i j}: k=0, \ldots, N ; i=1,2,3 ; j=1,2,3\right\},
\end{aligned}
$$

are sets of integers, $N \leq 4, t_{0} \equiv t$, and the upper limits for the flow-time integrations are linear combinations of the other flow-time variables, $t_{r}^{\mathrm{up}}=t_{r}^{\mathrm{up}}\left(t_{0}, \ldots, t_{r-1}\right)$. Initially, all the $c_{i}$ are zero. It is helpful to introduce these parameters for the subsequent discussion though. The momenta $p_{4}, p_{5}, p_{6}$ are linear combinations of the integration momenta $p_{1}$, $p_{2}, p_{3}$. For the three-loop quantities considered in this paper, the flow time $t$ is the only dimensionful external scale. A generalization of this notation to a different number of loops and to additional external scales is straightforward.

\subsection{IBP reduction of flow-time loop integrals}

In the first step, we reduce all occurring integrals to so-called master integrals by employing integration-by-parts (IBP) identities [33, 34]. They are based on the observation that integrals over total derivatives vanish in dimensional regularization. Applying the operator

$$
\mathcal{D}_{i j} \equiv \frac{\partial}{\partial p_{i}} \cdot p_{j}=\delta_{i j} D+p_{j} \cdot \frac{\partial}{\partial p_{i}}
$$

to the integrand of $I(t, \mathbf{n}, \mathbf{a}, \mathbf{c}, D)$ in eq. (3.3) thus results in a vanishing integral for all $i, j \in\{1,2,3\}$. On the other hand, explicitly acting with the derivative on the integrand leads to a sum of integrals where some of the indices $n_{i}$ and $c_{r}$ are shifted by $\pm 1 .{ }^{5}$ Repeated application of the $\mathcal{D}_{i j}$ thus leads to linear relations among integrals with different $\mathbf{n}$ and c. However, the number of flow-time integrations and the exponential function in the integrand still remain unaltered by this procedure.

\footnotetext{
${ }^{5}$ To be precise: at most one $n_{i}$ is shifted by -1 and at most one of the $n_{i}$ and at most one of the $c_{r}$ are shifted by +1 .
} 
We may apply an analogous strategy for the flow-time parameters though. Inserting a derivative with respect to one of the flow-time integration variables, one arrives at the sum of two integrals with fewer flow-time integrations and an altered exponential,

$$
\int_{0}^{t_{r}^{\mathrm{up}}} \mathrm{d} t_{r} \frac{\partial}{\partial t_{r}} f\left(t_{r}, \ldots\right)=f\left(t_{r}^{\mathrm{up}}, \ldots\right)-f(0, \ldots)
$$

On the other hand, explicit evaluation of the derivative at the integrand level either reduces one of the indices $c_{k}$ or one of the indices $n_{i}$ by one. One therefore arrives at linear relations among integrals with different $\mathbf{n}, \mathbf{c}, \mathbf{a}$, and different number of flow-time integrations $N$.

Applying the above operations to "seed integrals", i.e., integrals with fixed numerical values for the $\mathbf{n}, \mathbf{c}$, and $\mathbf{a}$, allows one to build a system of linear relations among the $I(t, \mathbf{n}, \mathbf{a}, \mathbf{c}, D)$. Defining an ordering (complexity) criterion allows one to solve the system using a Gaussian elimination type reduction for a minimal set of integrals (master integrals) [35]. Through this procedure all integrals of the type defined in eq. (3.3) are reduced to the minimal set of master integrals, which are simplest by the ordering criterion introduced. The system of linear relations is process dependent and for the observables in this study we construct it in Mathematica [36]. We use the specialized software Kira [37, 38] for the reduction of such linear equation systems to solve it.

While Kira alone is sufficient for the reduction of all our integrals at the two-loop level (see e.g. ref. [21]), we find that the algebraic solution of the system at the three-loop level would require more than $750 \mathrm{GiB}$ of RAM and thus exceeds our available computing resources. ${ }^{6}$ Using finite field and reconstruction techniques, which over the last decade have gained an increased use for higher-order perturbative calculations (see, e.g. refs. [39-41]), one can improve on the required computational resources as follows.

Since $t$ is the only dimensionful scale and can thus be factored out, the coefficients of the integrals in the linear system are simply polynomials in $D$. The individual steps for the reduction of the linear system only involve elementary arithmetic operations, which means that the coefficients of the master integrals are rational functions in $D$. One can then solve the linear system numerically over a finite field using a sufficiently large number of different integer values for $D$ ("probes") and reconstruct the rational functions in $D$ exactly. With this approach the size of the coefficients remain simple numbers and the requirements on computational resources can be improved.

While Kira already uses pyRed as a first step to remove linearly dependent equations over a finite field, in our approach pyRed is used to reduce the system itself multiple times over a finite field. The resulting numbers are processed with the library FireFly [42], which provides an efficient implementation of interpolation [43, 44] and rational-reconstruction algorithms $[45,46] .{ }^{7}$ In our case, we require 201 probes, chosen from three different finite fields, defined as prime fields $\mathbb{Z}_{p}$ with $p$ a 63-bit prime number, to reconstruct all coefficients, plus one additional probe in a fourth prime field to verify the reconstruction. For each probe, the solution of the system with Kira now just requires about $70 \mathrm{GiB}$ of RAM and takes about three CPU hours. In total, the reduction took less than three days, using ten threads on two Intel Xeon Gold 6138 processors.

\footnotetext{
${ }^{6}$ This calculation was performed before the release of Kira 1.2 [38]. It is well possible that these statements could change with the newly implemented features.

${ }^{7}$ We remark that FireFly also works for multi-variate rational functions.
} 
For the observables considered further below in this paper at the three-loop level we start with a total of 3195 integrals and can reduce them to a minimal set of 188 master integrals. ${ }^{8}$ Their numerical evaluation is described in the next section.

\subsection{Numerical computation of flow-time loop integrals}

As a first step of the numerical evaluation of the gradient-flow integrals given by eq. (3.3), we express the propagators through Schwinger-parameter integrals and map them from $x \in(0, \infty)$ to $y \in(0,1)$ using the simple transformation $x=y /(1-y)$. Momentum integrations are performed as $D$-dimensional Gaussian integrals after a diagonalization. Similarly, all flow-time integrations are mapped to the unit interval with simple linear transformations. The overall result is an integral over a unit hypercube.

The integrand typically involves a number of (overlapping) singularities, which we factorize using FIESTA [47], an implementation of the sector decomposition algorithm [48]. As opposed to regular Feynman integrals where sector decomposition is typically employed in combination with Feynman parameterization, it appears that we cannot restrict the singularities to the lower integration bound only. ${ }^{9}$ If an integral involves singularities both at the lower and the upper bound, we split all integration intervals in the middle, and map the singularities to the lower bound by an appropriate change of variable.

The sector decomposed integrals obtained from FIESTA are then integrated with our implementation of fully symmetric integration rules of order 13 [17, 50], which can handle integrable logarithmic-like singularities at the integration boundaries very well. We perform all arithmetic with 256-bit precision using the MPFR library [51], and used a local adaptive bisection in the direction of the largest fourth difference. A high precision arithmetic turns out to be necessary to achieve a relative numerical accuracy of $10^{-10}$ or better. The integration uncertainty is estimated by the difference between the integration results of rules of order 13 and 11 .

As a check of our results further below, we apply the numerical integration method to our unreduced set of 3195 integrals as well as to the set of 188 master integrals. Finding full agreement for the results obtained with both sets within numerical uncertainties serves as a check of the numerical integration as well as for the reduction procedure.

Analytical computation. For some master integrals we can find analytical results either through elementary methods with the help of Mathematica [36] or by using HyperInt [52]. The integration with Mathematica sometimes yields hypergeometric functions which can be expanded with the package HypExp [53, 54]. For example as shown further below, we find analytical results for all contributions which contain at least one factor of $T_{\mathrm{R}}$ in the color structure. A fully analytical calculation of integrals with other color factors requires a more detailed investigation. However, for all practical purposes, the numerical results provided in this paper are (more than) sufficient.

\footnotetext{
${ }^{8}$ When our observables are expressed in terms of the master integrals, the dependence of six of them drops out.

${ }^{9}$ To identify cases where singularities appear at the upper bound, we determine the degree of divergence for each subset of integration variables at the level of the Schwinger parameterization [49].
} 


\section{Observables}

Among the simplest quantities one can consider within the GFF are vacuum expectation values of gauge-invariant operators at finite flow time. It is one of the remarkable properties of the GFF that these operators do not require any renormalization beyond that of regular QCD, and that of the involved flowed fields. For the gauge coupling and the quark masses, the $\overline{\mathrm{MS}}$ renormalization is given by the replacement

$$
g \rightarrow g_{0} \equiv\left(\frac{\mu \mathrm{e}^{\gamma_{\mathrm{E}} / 2}}{\sqrt{4 \pi}}\right)^{\epsilon} Z_{g}\left(\alpha_{\mathrm{s}}(\mu)\right) g(\mu), \quad m_{f} \rightarrow m_{f, 0} \equiv Z_{m}\left(\alpha_{\mathrm{s}}(\mu)\right) m_{f}(\mu)
$$

in eq. (2.12), where $\mu$ is the renormalization scale, $\alpha_{\mathrm{s}}=g^{2} /(4 \pi)$, and $\gamma_{\mathrm{E}}=0.5772 \ldots$ the Euler-Mascheroni constant. Through the perturbative order required in this paper, the renormalization constants are given by

$$
\begin{aligned}
& Z_{g}\left(\alpha_{\mathrm{s}}\right)=1-\frac{\alpha_{\mathrm{s}}}{4 \pi} \frac{\beta_{0}}{2 \epsilon}+\left(\frac{\alpha_{\mathrm{s}}}{4 \pi}\right)^{2}\left(\frac{3 \beta_{0}^{2}}{8 \epsilon^{2}}-\frac{\beta_{1}}{4 \epsilon}\right)+\mathcal{O}\left(\alpha_{\mathrm{s}}^{3}\right), \\
& Z_{m}\left(\alpha_{\mathrm{s}}\right)=1-\frac{\alpha_{\mathrm{s}}}{4 \pi} \frac{\gamma_{m, 0}}{2 \epsilon}+\left(\frac{\alpha_{\mathrm{s}}}{4 \pi}\right)^{2}\left[\frac{1}{\epsilon^{2}}\left(\frac{\gamma_{m, 0}^{2}}{8}+\frac{\beta_{0} \gamma_{m, 0}}{4}\right)-\frac{\gamma_{m, 1}}{4 \epsilon}\right]+\mathcal{O}\left(\alpha_{\mathrm{s}}^{3}\right),
\end{aligned}
$$

with

$$
\begin{aligned}
\beta_{0} & =\frac{11}{3} C_{\mathrm{A}}-\frac{4}{3} T_{\mathrm{F}}, & \beta_{1} & =\frac{34}{3} C_{\mathrm{A}}^{2}-\left(4 C_{\mathrm{F}}+\frac{20}{3} C_{\mathrm{A}}\right) T_{\mathrm{F}}, \\
\gamma_{m, 0} & =6 C_{\mathrm{F}}, & \gamma_{m, 1} & =\frac{97}{3} C_{\mathrm{A}} C_{\mathrm{F}}+3 C_{\mathrm{F}}^{2}-\frac{20}{3} C_{\mathrm{F}} T_{\mathrm{F}} .
\end{aligned}
$$

$C_{\mathrm{F}}$ and $C_{\mathrm{A}}$ are the quadratic Casimir eigenvalues of the fundamental and the adjoint representation of the gauge group, respectively. Furthermore, $T_{\mathrm{F}}=T_{\mathrm{R}} n_{\mathrm{F}}$, with $n_{\mathrm{F}}$ the number of quark flavors, and $T_{\mathrm{R}}$ the trace normalization in the fundamental representation. For $\mathrm{SU}\left(N_{\mathrm{c}}\right)$, it is $C_{\mathrm{F}}=\left(N_{\mathrm{c}}^{2}-1\right) /\left(2 N_{\mathrm{c}}\right), C_{\mathrm{A}}=N_{\mathrm{c}}$, and $T_{\mathrm{R}}=1 / 2$.

The flowed gauge field $B_{\mu}^{a}(t, x)$ does not require renormalization, so that for example matrix elements of the gluon action density,

$$
E(t, x) \equiv \frac{1}{4} G_{\mu \nu}^{a}(t, x) G_{\mu \nu}^{a}(t, x),
$$

are finite after just the renormalization of $g$ and $m_{f}$. This allows for a direct comparison of results obtained in different regularization schemes (lattice and perturbation theory, for example).

On the contrary, flowed quark fields require a renormalization factor $Z_{\chi}^{1 / 2}\left(\alpha_{\mathrm{s}}\right)$ in order to render Green's functions finite. In the $\overline{\mathrm{MS}}$ scheme, it is $[3,21]$

$$
Z_{\chi}\left(\alpha_{\mathrm{s}}\right)=1-\frac{\alpha_{\mathrm{s}}}{4 \pi} \frac{\gamma_{\chi, 0}}{2 \epsilon}+\left(\frac{\alpha_{\mathrm{s}}}{4 \pi}\right)^{2}\left[\frac{1}{\epsilon^{2}}\left(\frac{\gamma_{\chi, 0}^{2}}{8}+\frac{\beta_{0} \gamma_{\chi, 0}}{4}\right)-\frac{\gamma_{\chi, 1}}{4 \epsilon}\right]+\mathcal{O}\left(\alpha_{\mathrm{s}}^{3}\right),
$$

with

$$
\begin{aligned}
& \gamma_{\chi, 0}=6 C_{\mathrm{F}}, \\
& \gamma_{\chi, 1}=\left(\frac{223}{3}-16 \ln 2\right) C_{\mathrm{A}} C_{\mathrm{F}}-(3+16 \ln 2) C_{\mathrm{F}}^{2}-\frac{44}{3} C_{\mathrm{F}} T_{\mathrm{F}} .
\end{aligned}
$$


The quantity

$$
S(t, x) \equiv Z_{\chi} \sum_{f=1}^{n_{\mathrm{F}}} \bar{\chi}_{f}(t, x) \chi_{f}(t, x)
$$

thus acquires an anomalous dimension, which prevents a direct comparison of results from different regularization schemes. Alternatively, one may work with "ringed quark fields" [20], which amounts to using

$$
\stackrel{\circ}{Z}_{\chi}(t, \mu)=-\frac{2 N_{\mathrm{c}} n_{\mathrm{F}}}{(4 \pi t)^{2}}\langle R(t)\rangle^{-1}, \quad \text { with } \quad R(t, x)=\sum_{f=1}^{n_{\mathrm{F}}} \bar{\chi}_{f}(t, x) \overleftrightarrow{\not D}^{\mathrm{F}} \chi_{f}(t, x)
$$

instead of $Z_{\chi}$ in order to renormalize the quark fields, where

$$
\overleftrightarrow{\mathcal{D}}^{\mathrm{F}}=\mathcal{D}^{\mathrm{F}}-\overleftarrow{\mathcal{D}}^{\mathrm{F}}
$$

This corresponds to a "physical" renormalization scheme, which means that the anomalous dimension of the operator

$$
\stackrel{\circ}{S}(t, x)=\zeta_{\chi}(t, \mu) S(t, x), \quad \text { with } \quad \zeta_{\chi}(t, \mu) \equiv Z_{\chi}^{-1} \stackrel{\circ}{Z}_{\chi}(t, \mu)
$$

vanishes. In eq. (4.8), we used the notation

$$
\langle\mathcal{O}(t)\rangle \equiv \int \mathrm{d}^{4} x\langle\mathcal{O}(t, x)\rangle
$$

for the zero-momentum Fourier transform of the vacuum expectation value of an operator $\mathcal{O}(t, x)$, which we adopt in the following.

The Feynman rules for the operators $E(t, x), S(t, x)$, and $R(t, x)$ introduced above are obtained in the same manner as those described in section 2.3. Expressing them in terms of the flowed fields $B(t, x)$ and $\chi_{f}(t, x)$, the operator $E(t, x)$ results in a bilinear, trilinear, and a quartic gluon vertex as displayed in eqs. (A.17)-(A.19), $S(t, x)$ corresponds to the single bilinear quark vertex given in eq. (A.20), and $R(t, x)$ to the bilinear quark vertex of eq. (A.21) and the quark-gluon vertex of (A.22). Similar to the flow vertices, the lines attached to these vertices are depicted with dashed arrows, indicating that they can be both flow lines and propagators. In contrast to the flow vertices, the flow time of all lines in these vertices is directed towards the corresponding operator. Therefore, these vertices set the largest flow time in the Feynman diagrams.

Using the methods described in sections 2 and 3, we can calculate the vacuum expectation values of $E(t, x), S(t, x)$, and $R(t, x)$ through three loops. Sample diagrams for these quantities are shown in figure 2 . The number of diagrams in each case is given in table 1; recall, however, that this takes into account the re-writing of the four-gluon vertex into trilinear vertices as described in section 3. Not included in this number are diagrams with closed flow-line loops, but integrals with scale-less sub-loops are counted in. 


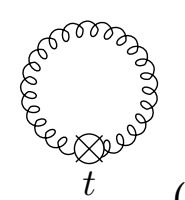

(a)

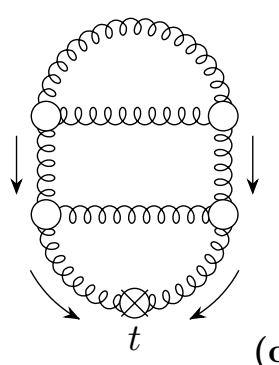

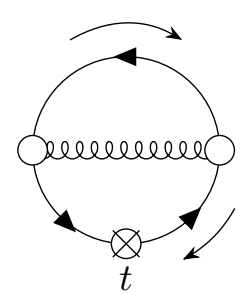

(b)

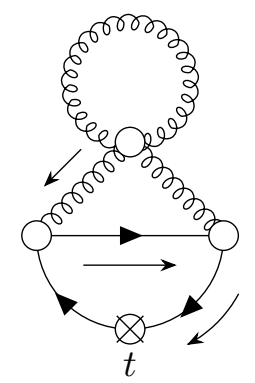

(e)

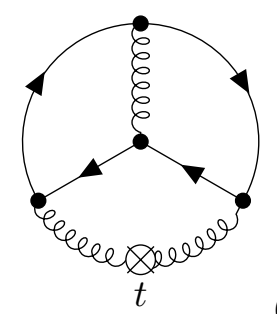

(c)

(d)

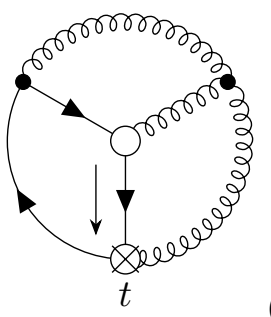

(f)

Figure 2. The only diagram contributing to $\langle E(t)\rangle$ at one loop (a); a two-loop diagram for $\langle S(t)\rangle$ and $\langle R(t)\rangle$ (b); and four three-loop diagrams: two diagrams for $\langle E(t)\rangle(\mathrm{c}, \mathrm{d})$; one diagram for $\langle S(t)\rangle$ and $\langle R(t)\rangle$ (e); and one diagram for $\langle R(t)\rangle(\mathrm{f})$.

\begin{tabular}{|c|c|c|c|c|}
\hline \#loops & 1 & 2 & 3 & $3\left(T_{\mathrm{R}}=0\right)$ \\
\hline$\langle E(t)\rangle$ & 1 & 11 & 232 & 211 \\
\hline$\langle S(t)\rangle$ & 1 & 8 & 210 & 202 \\
\hline$\langle R(t)\rangle$ & 1 & 11 & 311 & 300 \\
\hline
\end{tabular}

Table 1. The number of Feynman diagrams contributing to the quantities computed in this section. At three-loop level, the number for "quenched QCD" (marked as $T_{\mathrm{R}}=0$ ) is shown separately.

\subsection{Results for the gluon condensate at three loops}

We write the perturbative result for the gluon condensate as

$$
\langle E(t)\rangle=\frac{3 \alpha_{\mathrm{s}}}{4 \pi t^{2}} \frac{N_{\mathrm{A}}}{8}\left[e_{0}+\frac{\alpha_{\mathrm{s}}}{4 \pi} e_{1}+\left(\frac{\alpha_{\mathrm{s}}}{4 \pi}\right)^{2} e_{2}+\mathcal{O}\left(\alpha_{\mathrm{s}}^{3}\right)\right]+\mathcal{O}(m),
$$

where $\alpha_{\mathrm{s}}=\alpha_{\mathrm{s}}(\mu)$, with $\mu$ the renormalization scale. As indicated, quark mass terms are neglected here. Their effect at NLO has been studied in ref. [17] and it was found to be negligible. In this case, the $e_{i}$ depend only on the product $z \equiv \mu^{2} t$. For reasons that will become clear shortly, it is convenient to parameterize this dependence in terms of the variable

$$
L(z) \equiv \ln (2 z)+\gamma_{\mathrm{E}}
$$

A natural choice for the renormalization scale is defined by the inverse "smearing radius" $q_{8} \equiv 1 / \sqrt{8 t}$ of the gradient flow. However, from explicit higher order calculations, it was 
found that the slightly larger value

$$
\mu_{0}=\frac{e^{-\gamma_{\mathrm{E}} / 2}}{\sqrt{2 t}} \approx 1.5 q_{8}
$$

corresponding to $L\left(\mu_{0}^{2} t\right)=0$, improves the stability of the perturbative corrections [21] (see also ref. [17]). This was corroborated by the behavior of the $t \rightarrow 0$ extrapolation for thermodynamical quantities evaluated in ref. [22] using the two-loop result of ref. [21].

We thus write the coefficients of eq. (4.12) as

$$
\begin{aligned}
& e_{0}(z)=e_{0,0}, \quad e_{1}(z)=e_{1,0}+\beta_{0} L(z), \\
& e_{2}(z)=e_{2,0}+\left(2 \beta_{0} e_{1,0}+\beta_{1}\right) L(z)+\beta_{0}^{2} L^{2}(z),
\end{aligned}
$$

with $\beta_{0}, \beta_{1}$ from eq. (4.3). For the coefficients $e_{i, j}$, we find

$$
\begin{aligned}
& e_{0,0}=1, \quad e_{1,0}=\left(\frac{52}{9}+\frac{22}{3} \ln 2-3 \ln 3\right) C_{\mathrm{A}}-\frac{8}{9} T_{\mathrm{F}} \\
& e_{2,0}=27.9786 C_{\mathrm{A}}^{2}-(31.5652 \ldots) T_{\mathrm{F}} C_{\mathrm{A}}+\left(16 \zeta(3)-\frac{43}{3}\right) T_{\mathrm{F}} C_{\mathrm{F}}+\left(\frac{8 \pi^{2}}{27}-\frac{80}{81}\right) T_{\mathrm{F}}^{2},
\end{aligned}
$$

where $\zeta(z)$ is Riemann's $\zeta$ function with $\zeta(3)=1.20206 \ldots$. The three dots in the coefficient of $T_{\mathrm{F}} C_{\mathrm{A}}$ indicate that we were able to obtain an expression in analytical form. It can be found in appendix B, eq. (B.1). Only four decimal places of the numerical result for the $C_{\mathrm{A}}^{2}$ coefficient are displayed here, while our estimate of the numerical accuracy, obtained by propagating the uncertainty of the individual integrals to the final result, is about six digits beyond that. This estimate matches the observed differences between the numerical and analytical results in the cases where the latter have been computed. The same statements hold for the other observables listed below. The NLO coefficient $e_{1}$ was first evaluated in ref. [3]. Setting $\mu=q_{8}$, one finds that the NNLO result agrees with ref. [17] at the sub-percent level. ${ }^{10}$

The logarithmic terms $e_{n, k} L^{k}(z)$ obey the all-order recursion formula $(1 \leq k \leq n)$

$$
k e_{n, k}=\sum_{l=0}^{n-1}(n-l) e_{n-l-1, k-1} \beta_{l},
$$

which follows from renormalization-group invariance, i.e.

$$
\mu \frac{\mathrm{d}}{\mathrm{d} \mu}\langle E(t)\rangle=0, \quad \mu^{2} \frac{\mathrm{d}}{\mathrm{d} \mu^{2}} \alpha_{\mathrm{s}}=\alpha_{\mathrm{s}} \beta\left(\alpha_{\mathrm{s}}\right)=-\frac{\alpha_{s}^{2}}{4 \pi}\left(\beta_{0}+\frac{\alpha_{\mathrm{s}}}{4 \pi} \beta_{1}+\ldots\right) .
$$

This provides a welcome check of our calculation. The residual dependence on $\mu$ can be used as probe of the perturbative behavior. We refer to ref. [17] for such a study.

Since $\langle E(t)\rangle$ is also gauge independent, we are free to choose Feynman gauge for the QCD gauge parameter $(\xi=1)$ and $\kappa=1$ for the gradient-flow gauge parameter for the evaluation of the results presented above, which facilitates the actual calculation significantly.

\footnotetext{
${ }^{10}$ To be precise, all color coefficients of ref. [17] are compatible with our new calculation, except for the $C_{\mathrm{A}}^{2}$ term, for which "only" the first three digits agree, corresponding to an overly optimistic uncertainty estimate of ref. [17] for that term. The effect for any practical application should be irrelevant.
} 
However, we also check gauge invariance explicitly by evaluating the terms with the highest power in the gauge parameter $\xi$, i.e. $\xi^{4}$, and show that its coefficient vanishes. Indeed, this already happens algebraically after the reduction to master integrals, even before their numerical values are inserted. Keeping the gauge parameter fully general unfortunately leads to intermediate expressions which exceed our available computing resources.

\subsection{Results for the quark condensate at three loops}

To obtain the quark condensate with ringed fields we first consider the conversion factor $\zeta_{\chi}(t, \mu)$ from $\overline{\mathrm{MS}}$ renormalized to ringed quark fields as defined in eq. (4.10). Applying the methods described in section 2, we can calculate the diagrams contributing to the Green's function $\langle R(t)\rangle$ for massless quarks, which eventually leads to the result

$$
\begin{aligned}
\zeta_{\chi}(t, \mu)=1+ & \frac{\alpha_{\mathrm{s}}}{4 \pi}\left(\frac{\gamma_{\chi, 0}}{2} L\left(\mu^{2} t\right)-3 C_{\mathrm{F}} \ln 3-4 C_{\mathrm{F}} \ln 2\right) \\
+\left(\frac{\alpha_{\mathrm{s}}}{4 \pi}\right)^{2} & \left\{\frac{\gamma_{\chi, 0}}{4}\left(\beta_{0}+\frac{\gamma_{\chi, 0}}{2}\right) L^{2}\left(\mu^{2} t\right)+\left[\frac{\gamma_{\chi, 1}}{2}-\frac{\gamma_{\chi, 0}}{2}\left(\beta_{0}+\frac{\gamma_{\chi, 0}}{2}\right) \ln 3\right.\right. \\
& \left.\left.\quad-\frac{2}{3} \gamma_{\chi, 0}\left(\beta_{0}+\frac{\gamma_{\chi, 0}}{2}\right) \ln 2\right] L\left(\mu^{2} t\right)+C_{2}\right\}+\mathcal{O}\left(\alpha_{\mathrm{s}}^{3}, m\right) .
\end{aligned}
$$

The NLO result has been obtained in ref. [20], and the $\mu$-dependent NNLO terms were derived in ref. [21]. That paper also used a preliminary result for the coefficient $C_{2}$, derived for the first time in the current paper. Keeping four decimal places for the individual color coefficients, we find

$$
C_{2}=-23.7947 C_{\mathrm{A}} C_{\mathrm{F}}+30.3914 C_{\mathrm{F}}^{2}-(3.9226 \ldots) C_{\mathrm{F}} T_{\mathrm{F}},
$$

where again, as indicated by the three dots, the coefficient of $C_{\mathrm{F}} T_{\mathrm{F}}$ was obtained in analytical form and is given in appendix B, eq. (B.3).

Let us now turn to the quark condensate $\langle S(t)\rangle$. Since it vanishes in the chiral limit $m_{f}=0$, we compute the leading coefficient of the expansion in the quark masses $m_{f}$,

$$
\left\langle\zeta_{\chi} S(t)\right\rangle \equiv\langle\stackrel{\circ}{S}(t)\rangle=\sum_{f=1}^{n_{\mathrm{F}}} s_{f}(t)+\mathcal{O}\left(m^{2}\right),\left.\quad s_{f}(t) \equiv m_{f} \frac{\mathrm{d}\langle\stackrel{\circ}{S}(t)\rangle}{\mathrm{d} m_{f}}\right|_{m=0},
$$

where it is understood that all quark masses are set to zero after taking the derivative. This expansion can be applied before the loop and flow-time integrations are carried out, which means that, in the quark propagators, we write

$$
\frac{1}{\mathrm{i} q+m_{f}}=\frac{1}{\mathrm{i} \phi}+\frac{m_{f}}{q^{2}}+\mathcal{O}\left(m_{f}^{2}\right)
$$

and keep only the leading linear term in $m$ of the integrand. The resulting integrals are then again of the form shown in eq. (3.3). We thus write

$$
s_{f}(t)=-\frac{N_{\mathrm{c}} m_{f}}{8 \pi^{2} t}\left[s_{0}+\frac{\alpha_{\mathrm{s}}}{4 \pi} s_{1}+\left(\frac{\alpha_{\mathrm{s}}}{4 \pi}\right)^{2} s_{2}+\mathcal{O}\left(\alpha_{\mathrm{s}}^{3}\right)\right]+\mathcal{O}\left(m^{2}\right) .
$$


Renormalizing the quark masses in the $\overline{\mathrm{MS}}$ scheme according to eq. (4.1), we find

$$
\begin{aligned}
& s_{0}(z)=s_{0,0}, \quad s_{1}(z)=s_{1,0}+\frac{\gamma_{m, 0}}{2} L(z), \\
& s_{2}(z)=s_{2,0}+\frac{1}{2}\left[\left(\gamma_{m, 0}+2 \beta_{0}\right) s_{1,0}+\gamma_{m, 1}\right] L(z)+\frac{1}{8}\left(\gamma_{m, 0}+2 \beta_{0}\right) \gamma_{m, 0} L^{2}(z),
\end{aligned}
$$

with $z=\mu^{2} t$ and the $\gamma_{m, i}$ and $\beta_{i}$ from eq. (4.3). The non-logarithmic terms are given by

$$
\begin{aligned}
& s_{0,0}=1, \quad s_{1,0}=(4+4 \ln 2-3 \ln 3) C_{\mathrm{F}} \\
& s_{2,0}=19.6422 C_{\mathrm{F}}^{2}+41.3897 C_{\mathrm{F}} C_{\mathrm{A}}-(15.7975 \ldots) T_{\mathrm{F}} C_{\mathrm{F}},
\end{aligned}
$$

where again the $T_{\mathrm{F}} C_{\mathrm{F}}$ term is known analytically, as indicated by the three dots. It can be found in appendix B, eq. (B.4). The NLO term has been obtained in ref. [55], the NNLO term is new. Again, only four decimal places are displayed and our uncertainty estimate for the numerical integration is several digits beyond that.

The logarithmic terms $s_{n, k} L^{k}(z)$ obey the all-order recursion formula $(1 \leq k \leq n)$

$$
k s_{n, k}=\sum_{l=0}^{n-1}\left[\frac{\gamma_{n, l}}{2}+(n-l-1) \beta_{l}\right] s_{n-l-1, k-1},
$$

which follows from renormalization group invariance, i.e.

$$
\mu \frac{\mathrm{d}}{\mathrm{d} \mu} s_{f}(t)=0, \quad \mu \frac{\mathrm{d}}{\mathrm{d} \mu} m_{f}=m_{f} \gamma_{m}\left(\alpha_{\mathrm{s}}\right)=-m_{f} \frac{\alpha_{\mathrm{s}}}{4 \pi}\left(\gamma_{m, 0}+\frac{\alpha_{\mathrm{s}}}{4 \pi} \gamma_{m, 1}+\ldots\right)
$$

and the renormalization group equation for $\alpha_{\mathrm{s}}$ given in eq. (4.18). This again provides a welcome check of our calculation. The residual dependence on $\mu$ can be used as probe of the perturbative behavior. It is displayed in figure 3 for three values of the central energy scale $\mu_{0}=\left(2 t e^{\gamma_{\mathrm{E}}}\right)^{-1 / 2}$, see eq. (4.14). We observe a qualitatively similar behavior as was found for $\left\langle t^{2} E(t)\right\rangle$ in ref. [17]. While for large $\mu_{0}$, the reduction of the renormalization scale with increasing perturbative order is quite significant, this improvement reduces as $\mu_{0}$ gets closer to the non-perturbative regime. Taking the variation of $\mu$ by a factor of two around the central scale as an estimate of the uncertainty due to missing higher orders, one finds that they overlap between successive perturbative orders for all three values of $\mu_{0}$. However, the trend of the curves does not explicitly support $\mu_{0}$ as the central scale in this case, but seems to favor a slightly smaller scale.

Another important consistency check is that $\langle S(t)\rangle$ and $\langle R(t)\rangle$ can be renormalized with the same flowed-quark field renormalization constant $Z_{\chi}$ as obtained in ref. [21]. $\langle S(t)\rangle$ and $\langle R(t)\rangle$ are also gauge independent. For the calculation of the full NNLO result, we again chose Feynman gauge $(\xi=1)$ and $\kappa=1$. We check gauge invariance explicitly by evaluating the terms with the highest power in the gauge parameter $\xi$, which is $\xi^{3}$ for $\langle S(t)\rangle$ and $\langle R(t)\rangle$, and show that their coefficients vanish. This happens here also immediately after the reduction to master integrals. 


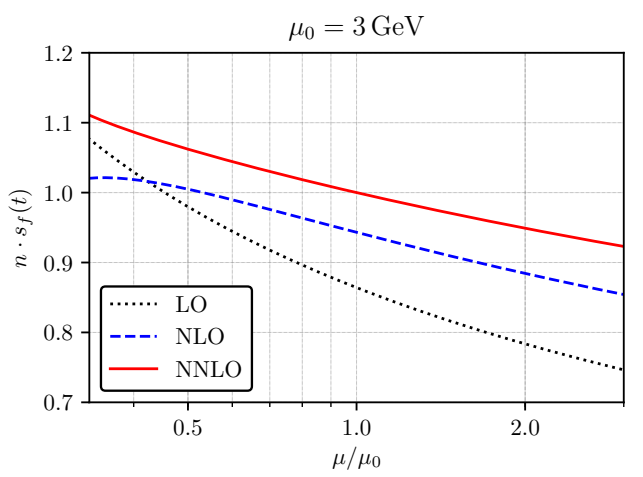

(a)

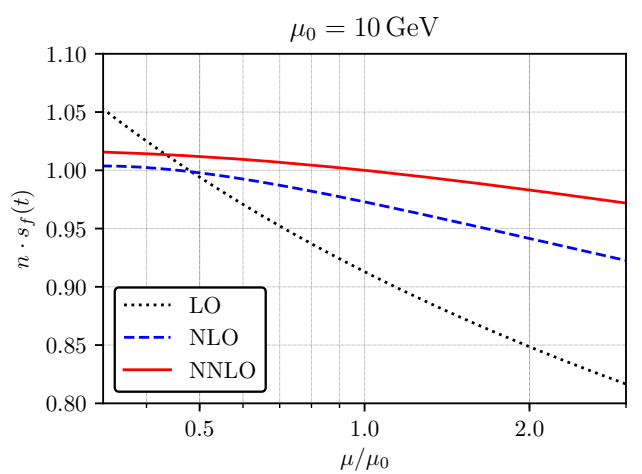

(b)

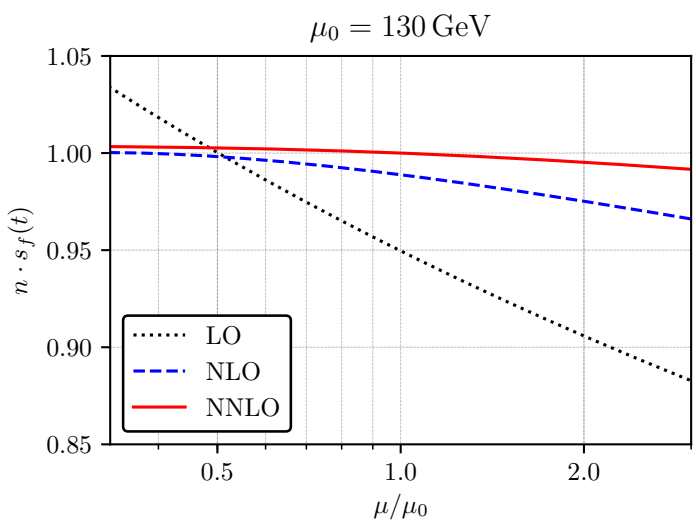

(c)

Figure 3. Renormalization scale dependence of $s_{f}(t)$, defined in eq. (4.21), for three different values of the central scale $\mu_{0}=e^{-\gamma_{\mathrm{E}} / 2} / \sqrt{2 t}$. (a) $\mu_{0}=3 \mathrm{GeV}$, corresponding to $t=(0.03 \mathrm{fm})^{2}$, where we use $n_{\mathrm{F}}=3$ and $\alpha_{\mathrm{s}}^{(3)}(3 \mathrm{GeV})=0.248$; (b) $\mu_{0}=10 \mathrm{GeV}\left(t=(0.01 \mathrm{fm})^{2}\right)$, where $n_{\mathrm{F}}=5$ and $\alpha_{\mathrm{s}}^{(5)}(10 \mathrm{GeV})=0.178 ;(\mathrm{c}) \mu_{0}=130 \mathrm{GeV}\left(t=\left(8 \cdot 10^{-4} \mathrm{fm}\right)^{2}\right)$, where $n_{\mathrm{F}}=5$ and $\alpha_{\mathrm{s}}^{(5)}(130 \mathrm{GeV})=0.112$. The running of $\alpha_{\mathrm{s}}(\mu)$ and $m_{f}(\mu)$ is evaluated with the help of RunDec $[56,57]$ at the corresponding loop order. All curves are normalized to the NNLO result at $\mu=\mu_{0}$.

\section{Gradient-flow coupling and mass}

\subsection{Gradient-flow coupling}

The renormalization group invariance of $\langle E(t)\rangle$ allows us to fix a scale $\mu=\sqrt{\rho / t}$ with constant $\rho \in \mathbb{R}$, and its proportionality to $\alpha_{\mathrm{s}}$ suggests to define a "gradient-flow coupling"

$$
\hat{\alpha}_{\rho}(\mu) \equiv \frac{32 \pi \rho^{2}}{3 N_{\mathrm{A}} \mu^{4}}\left\langle E\left(\rho / \mu^{2}\right)\right\rangle \equiv \alpha_{\mathrm{s}}(\mu) \sum_{n=0}^{\infty}\left(\frac{\alpha_{\mathrm{s}}(\mu)}{4 \pi}\right)^{n} e_{n}(\rho) .
$$

For the usual definition of the gradient-flow coupling [3], $\rho$ is set to $1 / 8=0.125$, while $\rho=1 /\left(2 e^{\gamma_{\mathrm{E}}}\right)=0.281 \ldots$ appears to be more suitable from a perturbative point of view (cf. eq. (4.14)). 
Perturbatively solving eq. (4.12) for $\alpha_{\mathrm{s}}$ results in

$$
\alpha_{\mathrm{s}}=\hat{\alpha}_{\rho}\left[1-e_{1}(\rho) \frac{\hat{\alpha}_{\rho}}{4 \pi}+\left(2 e_{1}^{2}(\rho)-e_{2}(\rho)\right)\left(\frac{\hat{\alpha}_{\rho}}{4 \pi}\right)^{2}+\ldots\right],
$$

with the coefficients $e_{n}(\rho)$ given in eq. (4.15). Such a transformation between mass-independent renormalization schemes leaves the first two coefficients of the $\beta$ function invariant, i.e.

$$
\begin{gathered}
\mu^{2} \frac{\mathrm{d}}{\mathrm{d} \mu^{2}} \hat{\alpha}_{\rho}(\mu)=\hat{\alpha}_{\rho}(\mu) \hat{\beta}_{\rho}\left(\hat{\alpha}_{\rho}\right), \\
\hat{\beta}_{\rho}\left(\hat{\alpha}_{\rho}\right)=-\sum_{n=0}^{\infty} \hat{\beta}_{\rho, n}\left(\frac{\hat{\alpha}_{\rho}}{4 \pi}\right)^{n}=-\frac{\hat{\alpha}_{\rho}}{4 \pi} \beta_{0}-\left(\frac{\hat{\alpha}_{\rho}}{4 \pi}\right)^{2} \beta_{1}-\sum_{n=2}^{\infty} \hat{\beta}_{\rho, n}\left(\frac{\hat{\alpha}_{\rho}}{4 \pi}\right)^{n},
\end{gathered}
$$

with $\beta_{0}$ and $\beta_{1}$ from eq. (4.3). The third coefficient is given by

$$
\hat{\beta}_{\rho, 2}=\beta_{2}-e_{1}(\rho) \beta_{1}+\left(e_{2}(\rho)-e_{1}^{2}(\rho)\right) \beta_{0},
$$

with the $\overline{\mathrm{MS}}$ coefficient $\beta_{2}$ which we quote here for the $\mathrm{SU}(3)$ gauge group:

$$
\beta_{2}=\frac{2857}{2}-\frac{5033}{18} n_{\mathrm{F}}+\frac{325}{54} n_{\mathrm{F}}^{2}
$$

\subsection{Gradient-flow mass}

Similarly, we may define a gradient-flow quark mass as

$$
\hat{m}_{f}^{(\rho)}(\mu)=-\left.\frac{8 \pi^{2} \rho}{N_{\mathrm{c}} \mu^{2}} m_{f} \frac{\mathrm{d}\left\langle\stackrel{\circ}{S}\left(\rho / \mu^{2}\right)\right\rangle}{\mathrm{d} m_{f}}\right|_{m=0}=m_{f}(\mu) \sum_{n=0}^{\infty} \hat{s}_{n}(\rho)\left(\frac{\hat{\alpha}_{\rho}(\mu)}{4 \pi}\right)^{n},
$$

where

$$
\hat{s}_{0}(\rho)=s_{0}(\rho), \quad \hat{s}_{1}(\rho)=s_{1}(\rho), \quad \hat{s}_{2}(\rho)=s_{2}(\rho)-s_{1}(\rho) e_{1}(\rho),
$$

with the $s_{n}$ and $e_{1}$ given in eqs. (4.24) and (4.15). The inverse relation is

$$
m_{f}=\hat{m}_{f}^{(\rho)}\left[1-\hat{s}_{1}(\rho) \frac{\hat{\alpha}_{\rho}}{4 \pi}+\left(\hat{s}_{1}^{2}(\rho)-\hat{s}_{2}(\rho)\right)\left(\frac{\hat{\alpha}_{\rho}}{4 \pi}\right)^{2}+\ldots\right] .
$$

The new mass obeys the renormalization group equation

$$
\mu \frac{\mathrm{d}}{\mathrm{d} \mu} \hat{m}_{f}^{(\rho)}(\mu)=\hat{\gamma}_{m}^{(\rho)}\left(\hat{\alpha}_{\rho}\right) \hat{m}_{f}^{(\rho)}(\mu) \equiv-\hat{m}_{f}^{(\rho)}(\mu) \sum_{n=0}^{\infty}\left(\frac{\hat{\alpha}_{\rho}(\mu)}{4 \pi}\right)^{n+1} \hat{\gamma}_{m, n}^{(\rho)}
$$

where

$$
\begin{aligned}
& \hat{\gamma}_{m, 0}^{(\rho)}=\gamma_{m, 0}, \quad \hat{\gamma}_{m, 1}^{(\rho)}=\gamma_{m, 1}-\gamma_{m, 0} e_{1}(\rho)+2 \beta_{0} \hat{s}_{1}(\rho), \\
& \hat{\gamma}_{m, 2}^{(\rho)}=\gamma_{m, 2}-2 \gamma_{m, 1} e_{1}(\rho)+\gamma_{m, 0}\left[2 e_{1}^{2}(\rho)-e_{2}(\rho)\right]+2 \beta_{1} \hat{s}_{1}(\rho)+2 \beta_{0}\left(2 \hat{s}_{2}(\rho)-\hat{s}_{1}^{2}(\rho)\right),
\end{aligned}
$$

with $\beta_{0}, \beta_{1}, \gamma_{m, 0}, \gamma_{m, 1}$ given in eq. (4.3), and $\gamma_{m, 2}$ the three-loop $\overline{\mathrm{MS}}$ coefficient which we quote for the $\mathrm{SU}(3)$ gauge group:

$$
\gamma_{m, 2}=2498-\left(\frac{4432}{27}+\frac{320}{3} \zeta(3)\right) n_{\mathrm{F}}-\frac{280}{81} n_{\mathrm{F}}^{2}
$$




\section{Conclusions}

A framework for the calculation of correlation functions in the perturbative gradient-flow formalism using tools and techniques from standard perturbative QCD calculations has been presented. It employs a Feynman-diagrammatic approach to the gradient-flow formalism, based on the five-dimensional field-theoretical formulation of ref. [16]. We implemented the corresponding QCD Feynman rules within the gradient-flow formalism as well as those of the composite operators relevant for the quantities considered in this paper, and automated the reduction and calculation of algebraic expressions and loop integrals to a large extent. An important ingredient of our setup is the reduction to master integrals with the help of integration-by-parts identities that also involve the flow-time variables. Except for a few cases where an analytic result was derived, the master integrals were evaluated numerically.

To demonstrate the viability of this setup, we reproduced and improved the threeloop result for the gluon condensate of an earlier calculation, which was based on explicit field-theoretic calculations at the level of Wick contractions [17].

Using this setup, we then evaluated the three-loop approximations to the quark condensate and the conversion factor from $\overline{\mathrm{MS}}$ renormalized to ringed quark fields, both of which are new results. Checks on gauge parameter independence, renormalization group invariance, as well as alternative calculational approaches (with and without reduction to master integrals) convinced us of the correctness of these results.

The gluon and the quark condensate lend themselves for straightforward definitions of a gradient-flow gauge coupling and a gradient-flow quark mass, for which we derive the three-loop matching relations to the $\overline{\mathrm{MS}}$ scheme. Provided that sufficiently accurate lattice results for these quantities become available, the conversion factors evaluated in this paper should allow for precise first-principle determinations of the gauge coupling, ${ }^{11}$ and possibly also quark masses.

While focusing on the calculation of vacuum matrix elements in this paper, our setup should be applicable in principle to more general problems which may involve a larger number of scales, for example.

\section{Acknowledgments}

We would like to thank Lucius Bushnaq and Yannick Kluth for their help in checking the Feynman rules and the general setup, Jonas Klappert for his support in using FireFly, Johann Usovitsch for his support in using Kira, and Alexander Voigt for general technical discussions. This work was supported by Deutsche Forschungsgemeinschaft (DFG) through project HA 2990/9-1, and by the U.S. Department of Energy under award No. DE-SC0008347. This document was prepared using the resources of the Fermi National Accelerator Laboratory (Fermilab), a U.S. Department of Energy, Office of Science, HEP User Facility. Fermilab is managed by Fermi Research Alliance, LLC (FRA), acting under Contract No. DE-AC02-07CH11359. Some of the calculations were performed with computing resources granted by RWTH Aachen University under project rwth0244. All Feynman diagrams in this paper were produced using TikZ-Feynman [59].

\footnotetext{
${ }^{11}$ First steps in this direction have been taken in ref. [58].
} 


\section{A Feynman rules}

In this section we present all the Feynman rules required for our calculation. All momenta are considered to be outgoing. $\xi$ is the usual QCD gauge parameter and $\kappa$ the additional gradient-flow gauge parameter introduced in the flow equations (2.2). Lines with a solid arrow are flow lines and lines with a dashed arrow can be both flow lines or flowed propagators. The symbols $p, q, r$ denote Euclidean four-momenta, while $s, t$ are real-valued flow-time variables.

\section{Propagators and flow lines.}

- gluon propagator:

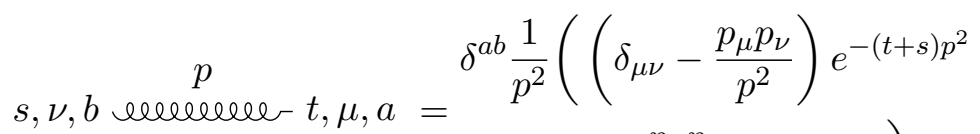

$$
\begin{aligned}
& \left.+\xi \frac{p_{\mu} p_{\nu}}{p^{2}} e^{-\kappa(t+s) p^{2}}\right)
\end{aligned}
$$

- (anti)quark propagator:

$$
s, \beta, j \longrightarrow \stackrel{p}{\longrightarrow} t, \alpha, i=\delta_{i j} \frac{(-\mathrm{i} \not p+m)_{\alpha \beta}}{p^{2}+m^{2}} e^{-(t+s) p^{2}}
$$

- ghost propagator:

$$
b \quad \begin{aligned}
& p \\
& b \quad a=\delta^{a b} \frac{1}{p^{2}}
\end{aligned}
$$

- gluon flow line:

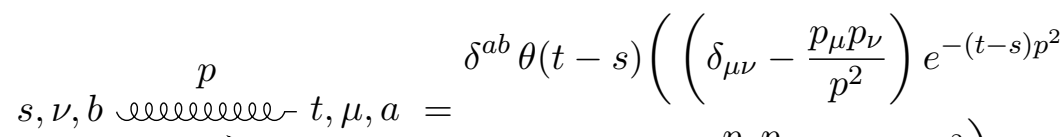

$$
\begin{aligned}
& \left.+\frac{p_{\mu} p_{\nu}}{p^{2}} e^{-\kappa(t-s) p^{2}}\right)
\end{aligned}
$$

- quark flow line:

$$
s, \beta, j \longrightarrow \stackrel{p}{\longrightarrow} t, \alpha, i=\delta^{i j} \delta_{\alpha \beta} \theta(t-s) e^{-(t-s) p^{2}}
$$

- antiquark flow line:

$$
s, \beta, j \stackrel{p}{\longrightarrow} t, \alpha, i=\delta^{i j} \delta_{\alpha \beta} \theta(t-s) e^{-(t-s) p^{2}}
$$




\section{Vertices.}

- three-gluon vertex:

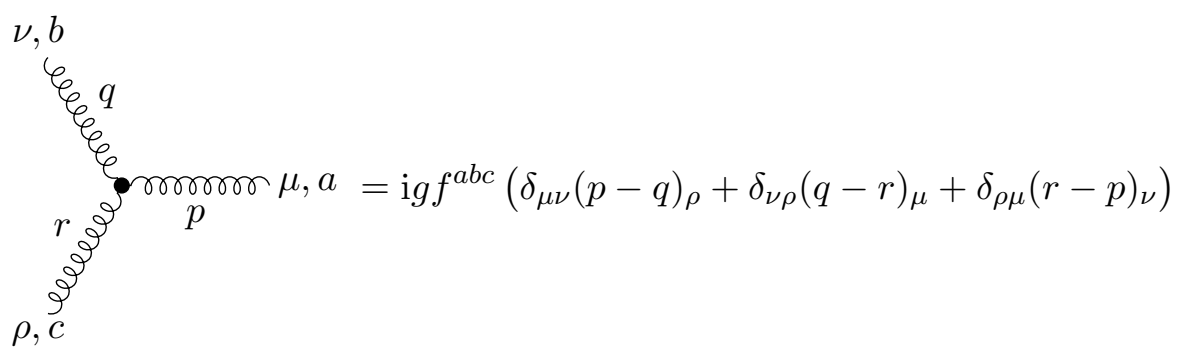

- four-gluon vertex:

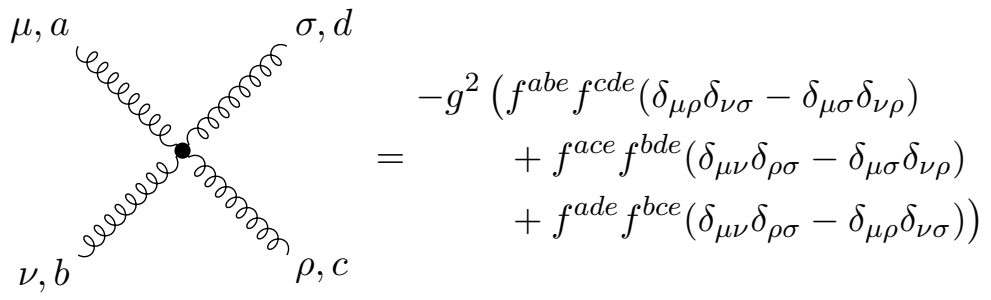

- ghost-gluon vertex:

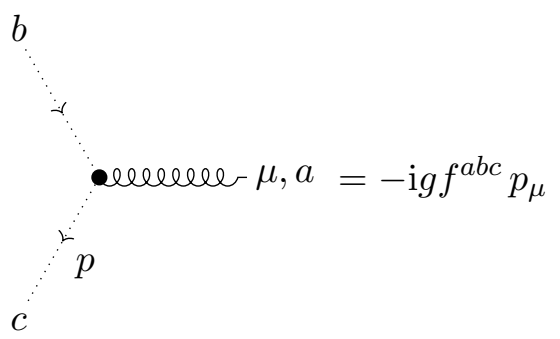

- quark-gluon vertex:

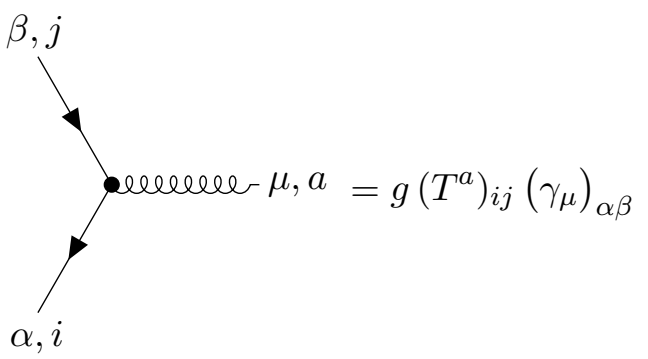


- two-plus-one gluon flow vertex:

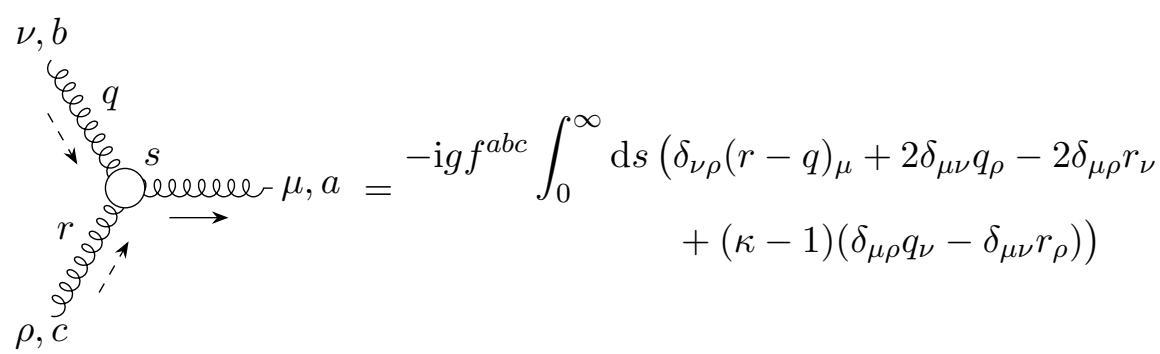

- three-plus-one gluon flow vertex:

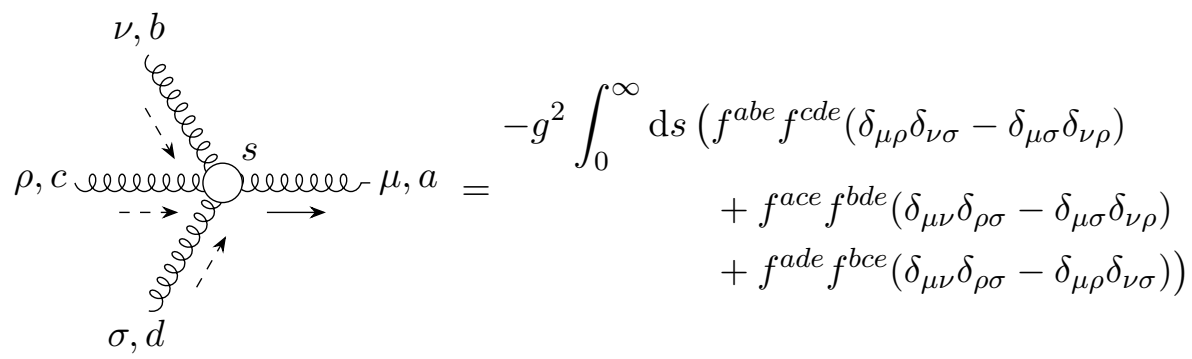

- quark-one-gluon flow vertex:

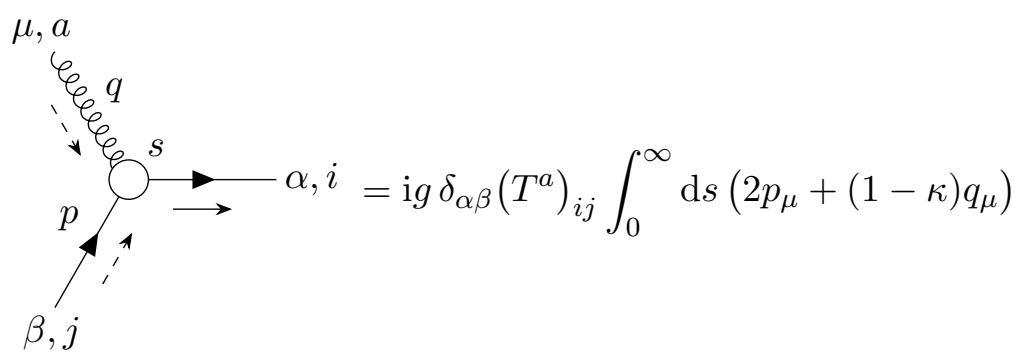

- antiquark-one-gluon flow vertex:

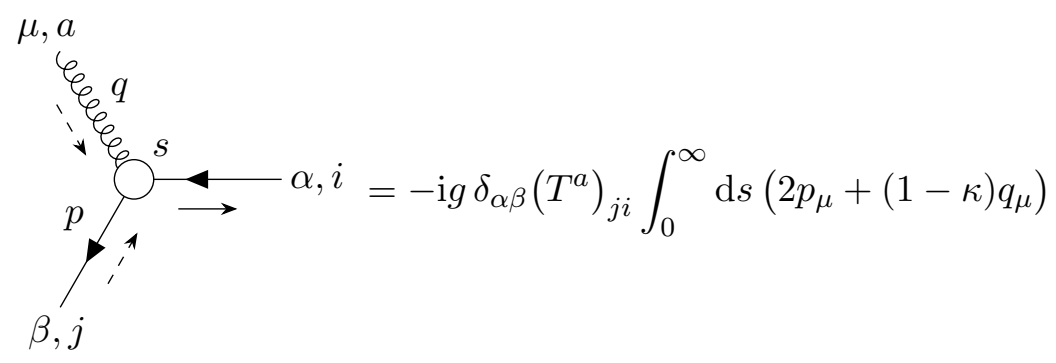


- quark-two-gluons flow vertex:

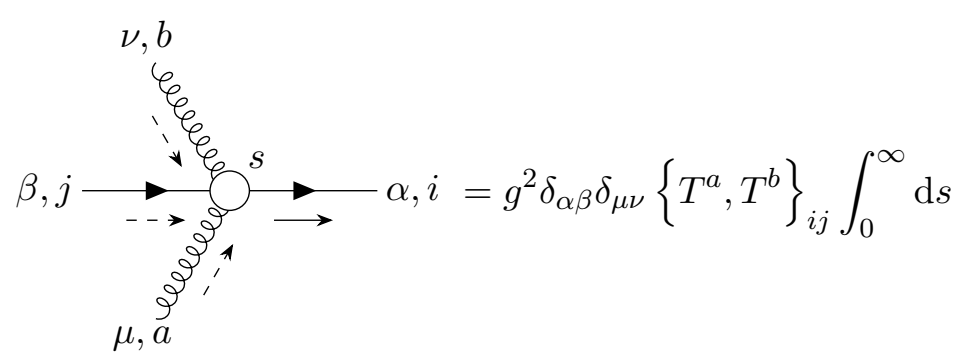

- antiquark-two-gluons flow vertex:

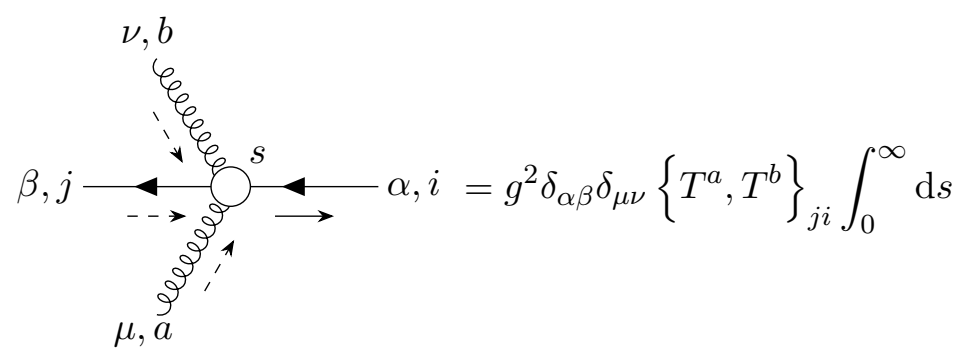

Feynman rules for the composite flow-time operators:

- $G_{\mu \nu}^{a}(t, x) G_{\mu \nu}^{a}(t, x)$ :

- two-gluon vertex:

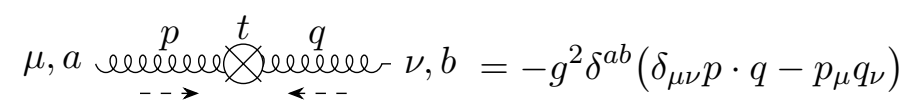

- three-gluon vertex:

$$
\begin{aligned}
& \mu, a \\
& \xi p
\end{aligned}
$$

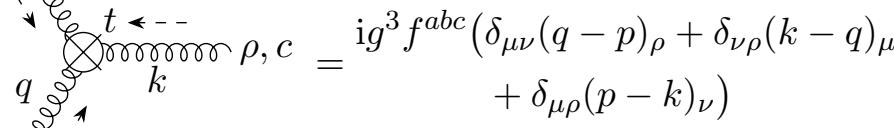

$$
\begin{aligned}
& \nu, b
\end{aligned}
$$

- four-gluon vertex:

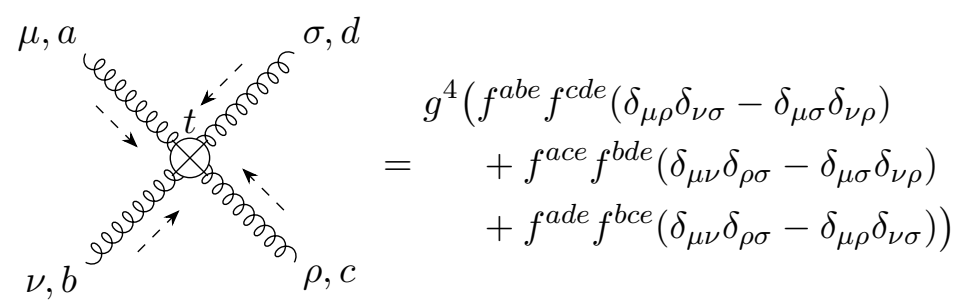


- $\bar{\chi}(t, x) \chi(t, x)$ :

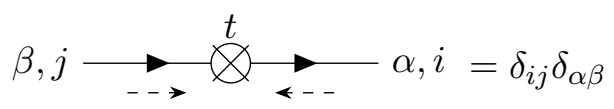

- $\bar{\chi}(t, x) \overleftrightarrow{\mathcal{D}}^{\mathrm{F}} \chi(t, x)$

- quark-antiquark vertex:

$$
\beta, j \longrightarrow \underset{\longrightarrow}{q} \stackrel{t}{\otimes} \underset{\leftrightarrow}{\stackrel{p}{\longrightarrow}} \alpha, i=\mathrm{i} \delta^{i j}(\not p-\not q)_{\alpha \beta}
$$

- quark-antiquark-gluon vertex:

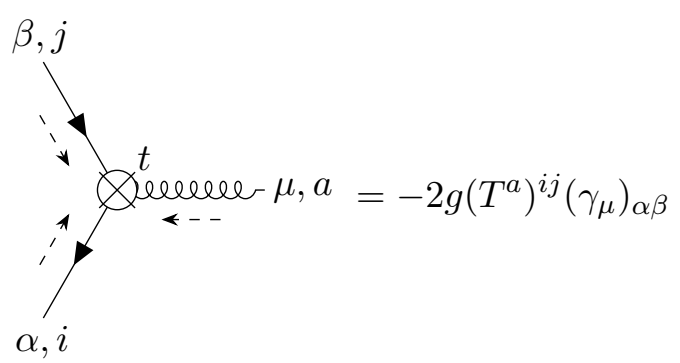

Auxiliary Feynman rules. The following Feynman rules are auxiliary Feynman rules for our implementation as described in section 3. They represent the vertices with four gluons or gluon-flow lines and allow us to factorise the color structure of all Feynman diagrams.

- four-gluon vertex:

$-\Sigma$ propagator:

$$
\rho, \sigma, b=========\mu, \nu, a=\delta^{a b} \delta_{\mu \rho} \delta_{\nu \sigma}
$$

- $\Sigma$-gluon-gluon vertex:

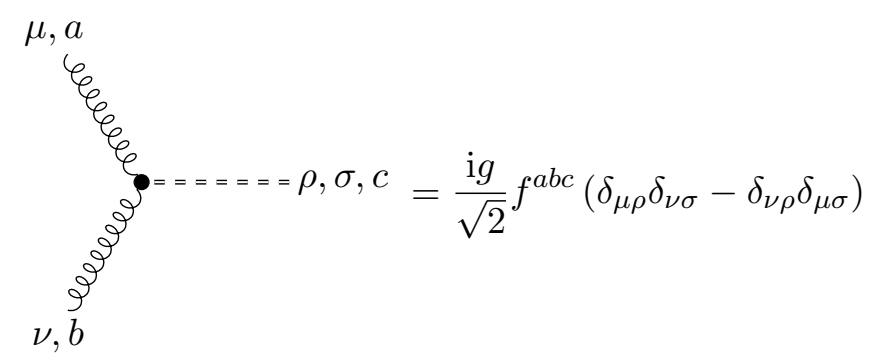


- three-plus-one gluon flow vertex:

$-\Sigma$ flow line:

$$
s, \rho, \sigma, b======t, \mu, \nu, a=\delta^{a b} \delta_{\mu \rho} \delta_{\nu \sigma} \delta(t-s)
$$

- outgoing $\Sigma$-gluon-gluon flow vertex:

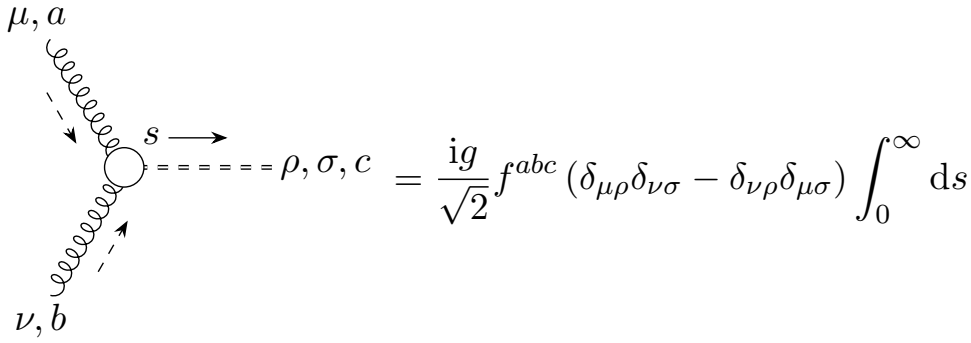

- incoming $\Sigma$-gluon-gluon flow vertex:

$$
\begin{aligned}
& \mu, a
\end{aligned}
$$

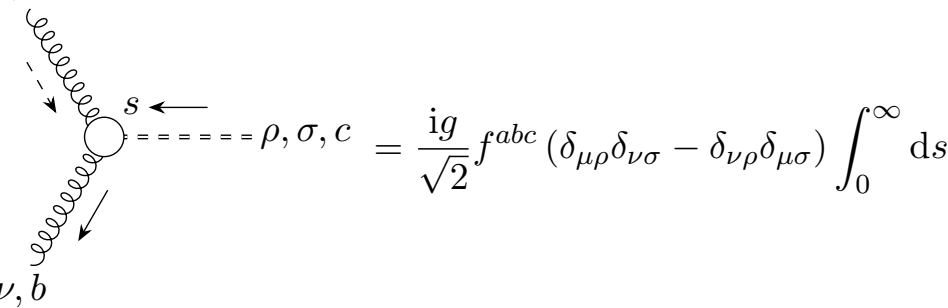

- four-gluon vertex from $G_{\mu \nu}^{a}(t, x) G_{\mu \nu}^{a}(t, x)$ :

$-\Sigma_{E}$ flow line:

$$
s, \rho, \sigma, b=t, \mu, \nu, a=\delta^{a b} \delta_{\mu \rho} \delta_{\nu \sigma} \delta(t-s)
$$

- outgoing $\Sigma_{E}$-gluon-gluon vertex:

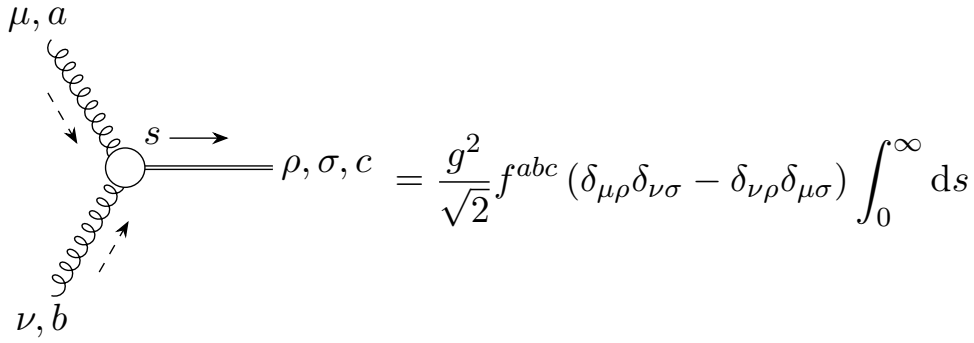

- incoming $\Sigma_{E}$-gluon-gluon vertex:

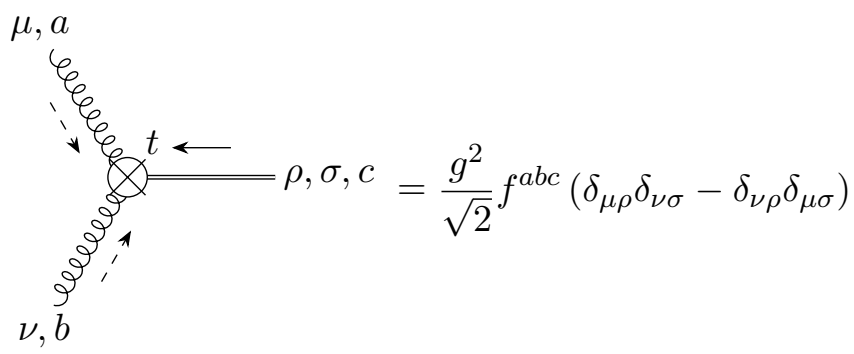




\section{B Analytical results}

Here, we provide the analytical results for the color coefficients as far as they are available. For the $T_{\mathrm{F}} C_{\mathrm{A}}$ term of $e_{2,0}$ in eq. (4.16), we find

$$
\begin{aligned}
-(31.5652 \ldots)= & \frac{2}{81}\left(-108 \mathrm{Li}_{3}\left(-\frac{1}{3}\right)-792 \mathrm{Li}_{2}\left(\frac{3}{4}\right)+216 \mathrm{Li}_{2}\left(-\frac{1}{3}\right)\right. \\
& +108 \mathrm{Li}_{1,2}\left(-3,-\frac{1}{3}\right)-216 \mathrm{Li}_{1,2}\left(1,-\frac{1}{3}\right)+216 \mathrm{Li}_{2,1}\left(-3,-\frac{1}{3}\right) \\
& -216 \mathrm{Li}_{2,1}\left(1,-\frac{1}{3}\right)+216 \mathrm{Li}_{1,1,1}\left(-1,-3,-\frac{1}{3}\right) \\
& -864 \mathrm{Li}_{1,1,1}\left(-1,3,-\frac{1}{3}\right)+216 \mathrm{Li}_{1,1,1}\left(1,3,-\frac{1}{3}\right) \\
& +432 \mathrm{Li}_{1,1,1}\left(3,1,-\frac{1}{3}\right)-1134 \zeta(3)+90 \pi^{2}-641-54 \ln ^{3} 3 \\
& +324 \ln ^{3} 2+324 \ln 2 \ln 23+270 \ln ^{2} 3-648 \ln 22 \ln 3-2232 \ln ^{2} 2 \\
& \left.+504 \ln 2 \ln 3-18 \pi^{2} \ln 3+1890 \ln 3+108 \pi^{2} \ln 2-2148 \ln 2\right),
\end{aligned}
$$

where

$$
\operatorname{Li}_{n_{1}, \ldots, n_{r}}\left(z_{1}, \ldots, z_{r}\right)=\sum_{0<k_{1}<\cdots<k_{r}} \frac{z_{1}^{k_{1}} \cdots z_{k}^{k_{r}}}{k_{1}^{n_{1}} \cdots k_{r}^{n_{r}}}
$$

are multiple polylogarithms $[60,61]$.

The $C_{\mathrm{F}} T_{\mathrm{F}}$ coefficient of $C_{2}$ in eq. (4.20) reads

$$
\begin{aligned}
-(3.9226 \ldots)= & -\frac{1}{18}\left(48 \operatorname{Li}_{2}\left(\frac{1}{9}\right)-2400 \operatorname{Li}_{2}\left(\frac{1}{3}\right)+672 \operatorname{Li}_{2}\left(\frac{3}{4}\right)-131+46 \pi^{2}\right. \\
& \left.+960 \ln ^{2} 2-1068 \ln ^{2} 3+1888 \ln 2-1032 \ln 3+624 \ln 2 \ln 3\right) .
\end{aligned}
$$

And finally, the $C_{\mathrm{F}} T_{\mathrm{F}}$ coefficient of $s_{2,0}$ in eq. (4.25) reads

$$
\begin{aligned}
-(15.7975 \ldots)= & \frac{16}{3} \mathrm{Li}_{2}\left(\frac{1}{9}\right)-\frac{800}{3} \mathrm{Li}_{2}\left(\frac{1}{3}\right)+104 \mathrm{Li}_{2}\left(\frac{3}{4}\right)-\frac{71}{6}+\frac{23 \pi^{2}}{9} \\
& +192 \ln ^{2} 2-\frac{362}{3} \ln ^{2} 3+\frac{544}{9} \ln 2-\frac{100}{3} \ln 3+\frac{56}{3} \ln 2 \ln 3 .
\end{aligned}
$$


Open Access. This article is distributed under the terms of the Creative Commons Attribution License (CC-BY 4.0), which permits any use, distribution and reproduction in any medium, provided the original author(s) and source are credited.

\section{References}

[1] R. Narayanan and H. Neuberger, Infinite $N$ phase transitions in continuum Wilson loop operators, JHEP 03 (2006) 064 [hep-th/0601210] [INSPIRE].

[2] M. Lüscher, Trivializing maps, the Wilson flow and the HMC algorithm, Commun. Math. Phys. 293 (2010) 899 [arXiv:0907.5491] [INSPIRE].

[3] M. Lüscher, Properties and uses of the Wilson flow in lattice QCD, JHEP 08 (2010) 071 [Erratum ibid. 03 (2014) 092] [arXiv:1006.4518] [INSPIRE].

[4] M. Lüscher, Future applications of the Yang-Mills gradient flow in lattice QCD, PoS (LATTICE2013) 016 (2014) [arXiv: 1308.5598] [INSPIRE].

[5] S. Borsányi et al., High-precision scale setting in lattice QCD, JHEP 09 (2012) 010 [arXiv:1203.4469] [INSPIRE].

[6] M. Lüscher, Chiral symmetry and the Yang-Mills gradient flow, JHEP 04 (2013) 123 [arXiv: 1302.5246] [INSPIRE].

[7] R. Sommer, Scale setting in lattice QCD, PoS (LATTICE2013)015 (2014) [arXiv:1401.3270] [INSPIRE].

[8] A. Ramos, The Yang-Mills gradient flow and renormalization, PoS (LATTICE2014) 017 (2015) [arXiv: 1506.00118] [INSPIRE].

[9] Flavour Lattice Averaging Group collaboration, FLAG Review 2019, arXiv: 1902.08191 [INSPIRE].

[10] S. Aoki, K. Kikuchi and T. Onogi, Geometries from field theories, PTEP 2015 (2015) 101B01 [arXiv: 1505.00131] [INSPIRE].

[11] S. Aoki, J. Balog, T. Onogi and P. Weisz, Flow equation for the large $N$ scalar model and induced geometries, PTEP 2016 (2016) 083B04 [arXiv:1605.02413] [INSPIRE].

[12] S. Aoki and S. Yokoyama, AdS geometry from CFT on a general conformally flat manifold, Nucl. Phys. B 933 (2018) 262 [arXiv:1709.07281] [InSPIRE].

[13] S. Aoki and S. Yokoyama, Flow equation, conformal symmetry and anti-de Sitter geometry, PTEP 2018 (2018) 031B01 [arXiv: 1707.03982] [INSPIRE].

[14] S. Aoki, J. Balog and S. Yokoyama, Holographic computation of quantum corrections to the bulk cosmological constant, PTEP 2019 (2019) 043 [arXiv: 1804.04636] [INSPIRE].

[15] S. Aoki, S. Yokoyama and K. Yoshida, Holographic geometry for non-relativistic systems emerging from generalized flow equations, Phys. Rev. D 99 (2019) 126002 [arXiv: 1902.02578] [INSPIRE].

[16] M. Lüscher and P. Weisz, Perturbative analysis of the gradient flow in non-abelian gauge theories, JHEP 02 (2011) 051 [arXiv:1101.0963] [INSPIRE].

[17] R.V. Harlander and T. Neumann, The perturbative QCD gradient flow to three loops, JHEP 06 (2016) 161 [arXiv:1606.03756] [INSPIRE]. 
[18] M. Dalla Brida and M. Lüscher, SMD-based numerical stochastic perturbation theory, Eur. Phys. J. C 77 (2017) 308 [arXiv: 1703.04396] [InSPIRE].

[19] H. Suzuki, Energy-momentum tensor from the Yang-Mills gradient flow, PTEP 2013 (2013) $083 \mathrm{~B} 03$ [Erratum ibid. 2015 (2015) 079201] [arXiv: 1304.0533] [INSPIRE].

[20] H. Makino and H. Suzuki, Lattice energy-momentum tensor from the Yang-Mills gradient flow - inclusion of fermion fields, PTEP 2014 (2014) 063B02 [Erratum ibid. 2015 (2015) 079202] [arXiv: 1403.4772] [INSPIRE].

[21] R.V. Harlander, Y. Kluth and F. Lange, The two-loop energy-momentum tensor within the gradient-flow formalism, Eur. Phys. J. C 78 (2018) 944 [arXiv: 1808.09837] [InSPIRE].

[22] T. Iritani, M. Kitazawa, H. Suzuki and H. Takaura, Thermodynamics in quenched QCD: energy-momentum tensor with two-loop order coefficients in the gradient-flow formalism, PTEP 2019 (2019) 023B02 [arXiv: 1812.06444] [INSPIRE].

[23] C. Monahan and K. Orginos, Quasi parton distributions and the gradient flow, JHEP 03 (2017) 116 [arXiv : 1612.01584] [INSPIRE].

[24] C. Monahan, Smeared quasidistributions in perturbation theory, Phys. Rev. D 97 (2018) 054507 [arXiv: 1710.04607] [INSPIRE].

[25] P. Nogueira, Automatic Feynman graph generation, J. Comput. Phys. 105 (1993) 279.

[26] P. Nogueira, Abusing qgraf, Nucl. Instrum. Meth. A 559 (2006) 220 [inSPIRE].

[27] K. Chetyrkin, private communication.

[28] R. Harlander, T. Seidensticker and M. Steinhauser, Corrections of $\mathcal{O}\left(\alpha \alpha_{s}\right)$ to the decay of the $Z$ boson into bottom quarks, Phys. Lett. B 426 (1998) 125 [hep-ph/9712228] [INSPIRE].

[29] T. Seidensticker, Automatic application of successive asymptotic expansions of Feynman diagrams, in 6th International Workshop on New Computing Techniques in Physics Research: Software Engineering, Artificial Intelligence Neural Nets, Genetic Algorithms, Symbolic Algebra, Automatic Calculation (AIHENP 99), Heraklion, Crete, Greece, April 12-16, 1999 (1999) [hep-ph/9905298] [INSPIRE].

[30] J.A.M. Vermaseren, New features of FORM, math-ph/0010025 [INSPIRE].

[31] J. Kuipers, T. Ueda, J.A.M. Vermaseren and J. Vollinga, FORM version 4.0, Comput. Phys. Commun. 184 (2013) 1453 [arXiv: 1203.6543] [INSPIRE].

[32] T. van Ritbergen, A.N. Schellekens and J.A.M. Vermaseren, Group theory factors for Feynman diagrams, Int. J. Mod. Phys. A 14 (1999) 41 [hep-ph/9802376] [InSPIRE].

[33] F.V. Tkachov, A Theorem on Analytical Calculability of Four Loop Renormalization Group Functions, Phys. Lett. 100B (1981) 65 [InSPIRE].

[34] K.G. Chetyrkin and F.V. Tkachov, Integration by Parts: The Algorithm to Calculate B-functions in 4 Loops, Nucl. Phys. B 192 (1981) 159 [INSPIRE].

[35] S. Laporta, High precision calculation of multiloop Feynman integrals by difference equations, Int. J. Mod. Phys. A 15 (2000) 5087 [hep-ph/0102033] [InSPIRE].

[36] Wolfram Research, Inc., Mathematica, Version 11.3, Champaign, IL (2018).

[37] P. Maierhöfer, J. Usovitsch and P. Uwer, Kira - A Feynman integral reduction program, Comput. Phys. Commun. 230 (2018) 99 [arXiv: 1705.05610] [INSPIRE].

[38] P. Maierhöfer and J. Usovitsch, Kira 1.2 Release Notes, arXiv:1812.01491 [InSPIRE]. 
[39] M. Kauers, Fast solvers for dense linear systems, Nucl. Phys. Proc. Suppl. 183 (2008) 245 [INSPIRE].

[40] P. Kant, Finding Linear Dependencies in Integration-By-Parts Equations: A Monte Carlo Approach, Comput. Phys. Commun. 185 (2014) 1473 [arXiv:1309.7287] [INSPIRE].

[41] T. Peraro, Scattering amplitudes over finite fields and multivariate functional reconstruction, JHEP 12 (2016) 030 [arXiv:1608.01902] [INSPIRE].

[42] J. Klappert and F. Lange, Reconstructing Rational Functions with FireFly, arXiv:1904.00009 [INSPIRE].

[43] R. Zippel, Interpolating Polynomials from their Values, Theor. Comp. Sci. 9 (1990) 375.

[44] A. Cuyt and W.-s. Lee, Sparse interpolation of multivariate rational functions, J. Symb. Comp. 412 (2011) 1445.

[45] P.S. Wang, A p-adic Algorithm for Univariate Partial Fractions, Proc. ACM Symp. Symbolic Algebraic Comp. 1981 (1981) 212.

[46] M. Monagan, Maximal Quotient Rational Reconstruction: An Almost Optimal Algorithm for Rational Reconstruction, Proc. Int. Symp. Symbolic Algebraic Comp. 2004 (2004) 243.

[47] A.V. Smirnov, FIESTA 3: cluster-parallelizable multiloop numerical calculations in physical regions, Comput. Phys. Commun. 185 (2014) 2090 [arXiv:1312.3186] [InSPIRE].

[48] T. Binoth and G. Heinrich, Numerical evaluation of multiloop integrals by sector decomposition, Nucl. Phys. B 680 (2004) 375 [hep-ph/0305234] [INSPIRE].

[49] E. Panzer, On hyperlogarithms and Feynman integrals with divergences and many scales, JHEP 03 (2014) 071 [arXiv: 1401.4361] [INSPIRE].

[50] A.C. Genz and A.A. Malik, An Imbedded Family of Fully Symmetric Numerical Integration Rules, SIAM J. Numer. Anal. 20 (1983) 580.

[51] L. Fousse, G. Hanrot, V. Lefèvre, P. Pélissier and P. Zimmermann, MPFR: A Multiple-precision Binary Floating-point Library with Correct Rounding, ACM Trans. Math. Softw. 33 (2007) 13.

[52] E. Panzer, Algorithms for the symbolic integration of hyperlogarithms with applications to Feynman integrals, Comput. Phys. Commun. 188 (2015) 148 [arXiv:1403.3385] [INSPIRE].

[53] T. Huber and D. Maître, HypExp: A Mathematica package for expanding hypergeometric functions around integer-valued parameters, Comput. Phys. Commun. 175 (2006) 122 [hep-ph/0507094] [INSPIRE].

[54] T. Huber and D. Maître, HypExp 2, Expanding Hypergeometric Functions about Half-Integer Parameters, Comput. Phys. Commun. 178 (2008) 755 [arXiv:0708.2443] [InSPIRE].

[55] H. Makino, O. Morikawa and H. Suzuki, Gradient flow and the Wilsonian renormalization group flow, PTEP 2018 (2018) 053B02 [arXiv: 1802.07897] [INSPIRE].

[56] K.G. Chetyrkin, J.H. Kuhn and M. Steinhauser, RunDec: A Mathematica package for running and decoupling of the strong coupling and quark masses, Comput. Phys. Commun. 133 (2000) 43 [hep-ph/0004189] [INSPIRE].

[57] F. Herren and M. Steinhauser, Version 3 of RunDec and CRunDec, Comput. Phys. Commun. 224 (2018) 333 [arXiv:1703.03751] [INSPIRE]. 
[58] E. Lambrou, Determining $\alpha_{s}$ by using the gradient flow in the quenched theory, PoS (LATTICE2016) 196 (2016) [INSPIRE].

[59] J. Ellis, TikZ-Feynman: Feynman diagrams with TikZ, Comput. Phys. Commun. 210 (2017) 103 [arXiv: 1601.05437] [INSPIRE].

[60] A.B. Goncharov, Multiple polylogarithms, cyclotomy and modular complexes, Math. Res. Lett. 5 (1998) 497 [arXiv:1105.2076] [INSPIRE].

[61] J.M. Borwein, D.M. Bradley, D.J. Broadhurst and P. Lisonek, Special values of multiple polylogarithms, Trans. Am. Math. Soc. 353 (2001) 907 [math/9910045] [INSPIRE]. 